\title{
GEM: a generic ecological model for estuaries and coastal waters
}

\author{
Anouk N. Blauw · Hans F. J. Los • \\ Marinus Bokhorst · Paul L. A. Erftemeijer
}

Received: 17 March 2008/Revised: 18 August 2008/Accepted: 24 August 2008/Published online: 23 September 2008

(C) Springer Science+Business Media B.V. 2008

\begin{abstract}
The set-up, application and validation of a generic ecological model (GEM) for estuaries and coastal waters is presented. This model is a comprehensive ecological model of the bottom of the foodweb, consisting of a set of modules, representing specific water quality processes and primary production that can be combined with any transport model to create a dedicated model for a specific ecosystem. GEM links different physical, chemical and ecological model components into one generic and flexible modelling tool that allows for variable sized, curvilinear grids to accomodate both the requirements for local accuracy while maintaining a relatively short model run-time. The GEM model describes the behaviour of nutrients, organic matter and primary producers in estuaries and coastal waters, incorporating dynamic process modules for dissolved oxygen, nutrients and phytoplankton. GEM integrates the best aspects of existing Dutch estuarine models that were mostly dedicated to only one type of ecosystem,
\end{abstract}

Handling editor: P. Viaroli

A. N. Blauw · H. F. J. Los · P. L. A. Erftemeijer $(\square)$ Deltares (formerly WL I Delft Hydraulics), PO Box 177, 2600 MH Delft, The Netherlands e-mail: paul.erftemeijer@deltares.nl

\section{Bokhorst}

Centre for Water Management (formerly National Institute for Coastal and Marine Management/RIKZ), Zuiderwagenplein 2, 8224 AD Lelystad, The Netherlands geographic area or subset of processes. Particular strengths of GEM include its generic applicability and the integration and interaction of biological, chemical and physical processes into one predictive tool. The model offers flexibility in choosing which processes to include, and the ability to integrate results from different processes modelled simultaneously with different temporal resolutions. The generic applicability of the model is illustrated using a number of representative examples from case studies in which the GEM model was successfully applied. Validation of these examples was carried out using the 'cost function' to compare model results with field observations. The validation results demonstrated consistent accuracy of the GEM model for various key parameters in both spatial dimensions (horizontally and vertically) as well as temporal dimensions (seasonally and across years) for a variety of water systems without the need for major reparameterisation.

Keywords Generic ecological model . GEM · Nutrients · Phytoplankton modelling · Validation · Water quality

\section{Introduction}

Growing stresses from conflicting human demands, anthropogenic impacts and climate change on coastal and estuarine environments (UNEP, 2006; Airoldi \& 
Beck, 2007), along with requirements from recent international legislative agreements (e.g. EU Water Framework Directive) to control and reduce undesirable ecosystem changes (Devlin et al., 2007), demand greater knowledge and understanding of the dynamics and driving forces of these complex water systems. Three-dimensional ecological models have the capacity to provide consistent distributions and dynamics of the lower trophic levels on their regional, annual and decadal scales, which cannot be derived to this degree of coverage by field monitoring observations (Moll \& Radach, 2003).

Estuarine and coastal waters pose a challenge to modellers, in terms of physics, biogeochemistry and ecology. Substantial river discharges and relatively shallow nearshore waters often result in large fluctuations and strong spatial gradients in salinity, suspended matter concentrations, nutrient concentrations and algal biomass in such water systems. These characteristics, along with complex benthic-pelagic interactions and light attentuation issues, have proven difficult to replicate in models (Radach \& Moll, 2006), in particular with regard to scales of temporal and spatial resolution required to simulate the possible impacts of future conditions, including management scenarios.

Over the past decades, a relatively large number of models have been developed for simulating nutrient cycles, primary production and ecosystem functioning in Dutch estuarine and coastal waters. Examples include MANS (Los et al., 1994), North Sea BLOOM (Los \& Bokhorst, 1997; Los \& Wijsman, 2007), SMOES (Klepper et al., 1994; Scholten \& Van der Tol, 1994), MOSES (Soetaert \& Herman, 1995a, b; Soetaert et al., 1994), ECOWASP (Brinkman, 1993) and ERSEM (Baretta et al., 1995). In addition, various model applications simulating aspects of ecosystem functioning of the North Sea (and adjacent Dutch coastal waters) have been developed at WL I Delft Hydraulics in response to specific management questions, often in close cooperation with the National Institute for Coastal and Marine Management (De Vries et al., 1998; Los \& Bokhorst, 1997; De Kok et al., 1995, 2001; Gerritsen et al., 2001; Delhez et al., 2004). These latter models typically have a relatively high spatial resolution, especially in the coastal zone, compared to most other North Sea models (Moll \& Radach, 2003), but they traditionally use relatively simple (if any) model formulations for food web interactions and organisms other than phytoplankton. This has been in contrast with most ecological models, which are usually detailed in ecological parameterization for specific ecological processes but lack spatial resolution in the underlying hydrodynamics and are mostly developed for application in only one geographic area. The ECOWASP model (Brinkman, 1993), for example, simulates the population ecology of mussels in the Wadden Sea at the level of size classes and year classes. The spatial resolution of such models, however, has been very low, ranging from 6 segments in the Wadden Sea (ECOWASP) to large ICES boxes, including the entire cross-shore gradient in Dutch coastal waters in only one model segment (ERSEM) (Baretta et al., 1995).

All of these models have proven useful tools in scientific research of estuarine ecosystems and for site-specific scenario studies of management strategies (Moll \& Radach, 2003). Most were, however, developed for a specific region, focussed on a specific area of expertise, differed markedly in model complexity and level of temporal and spatial resolution, and served different objectives. Typically, such individual process-oriented ecological models perform well for the particular water system for which they were developed, but when applied to other systems, their performance tends to be poor even after reparameterization (Fitz et al., 1996).

Therefore, the Dutch National Institute for Coastal and Marine Management (RIKZ) initiated the development of a Generic Ecological Model for estuaries (GEM), an integrated model that includes physical, chemical and ecological processes at a sufficient level of detail and in a consistent way. The goal of the generic ecological model was to integrate the best aspects of the existing (Dutch) models and expertise that are dedicated to one ecosystem or only a subset of relevant processes into an integrated coherent model that allows for general application to different coastal and estuarine systems.

The resulting GEM model has been applied for over a decade in a range of different consultancies and studies by WL I Delft Hydraulics, but the model set-up has not yet been scientifically published. A recent audit of the GEM model by an independent panel of international scientific experts (including Alain Menesguen, Paul Tett and William Silvert) strongly encouraged publication of the model (in 
particular, the approach for phytoplankton) and its promising results (Van de Wolfshaar, 2007).

The present paper describes the background, model set-up, application and validation of GEM. Our main objectives were to integrate biological and physical processes in a simulation of basic ecosystem dynamics for generic application to estuarine and coastal waters. The generic applicability of the model is illustrated using a number of representative examples from four selected case studies encompassing different spatial and temporal dimensions and a variety of different water systems in which the GEM model was successfully applied and validated.

\section{Description of the 'gem' model}

\section{Modelling environment}

GEM consists of a subset of process formulations from the process library of DELWAQ: the program for modelling water quality and aquatic ecology in the Delft3D modelling suite (WL I Delft Hydraulics, 2003). Delft3D-WAQ, Delft3D-ECO, Delft3D-SED and DBS are other subsets from the same process library that partly overlap with GEM. DELWAQ uses a finite grid approach. Sources and sinks of variables due to processes in the water are included in the advection-diffusion equation. A large selection of numerical schemes is available to solve the transport part in the advection-diffusion equation below. Processes (P) are all simulated with an explicit numerical scheme. ${ }^{1}$

$$
\begin{aligned}
\frac{\partial C}{\partial t}= & -u \frac{\partial C}{\partial x}-v \frac{\partial C}{\partial y}-w \frac{\partial C}{\partial z}+\frac{\partial}{\partial x}\left(D_{x} \frac{\partial C}{\partial x}\right) \\
& +\frac{\partial}{\partial y}\left(D_{y} \frac{\partial C}{\partial y}\right)+\frac{\partial}{\partial z}\left(D_{z} \frac{\partial C}{\partial z}\right)+S+P
\end{aligned}
$$

where

$$
C \text { : concentration }\left(\mathrm{g} \mathrm{m}^{-3}\right)
$$$$
\mathrm{u}, \mathrm{v}, \mathrm{w} \text { : components of the velocity vector }\left(\mathrm{m} \mathrm{s}^{-1}\right)
$$

\footnotetext{
${ }^{1}$ Numerical schemes used in the four case studies described in this paper were as follows: implicit upwind scheme with an iterative solver (Case 1, Veerse Meer); horizontal: FCT Scheme, vertical: implicit in time and central discretisation (Case 2, North Sea); flux correct transport (FCT) method (Case 3, Venice Lagoon); and horizontal upwind scheme, vertical: implicit in time and central discretisation (Case 4, Sea of Marmara).
}

$D_{\mathrm{x}}, D_{\mathrm{y}}, D_{\mathrm{z}}$ : components of the dispersion tensor $\left(\mathrm{m}^{2} \mathrm{~s}^{-1}\right)$

$\mathrm{x}, \mathrm{y}, \mathrm{z}$ : coordinates in three spatial dimensions (m) $\mathrm{S}$ : source and sinks of mass due to loads and boundaries

P: sources and sinks of mass due to processes

t: time (s)

The advection and diffusion fluxes between grid cells are usually derived from a hydrodynamic model (e.g. Delft3D-FLOW) for the same model area. DELWAQ has been used successfully for many different types of applications, including the simulation of dredging plumes, thermal discharges and various water pollution studies (Van Gils et al., 1993; Van der Molen et al., 1994; Ouboter et al., 1998; Van Gils, 1998).

Not all processes incorporated need the same level of detail with respect to time step and grid size. DELWAQ enables the use of different time steps and grids for different processes. The model will then aggregate and de-aggregate the input and output parameters of the processes. One can, for example, use a different time step for transport (tide resolving) and water quality processes, which in general show less steep gradients in both space and time. This way a considerable reduction of simulation time can be achieved with only a limited loss in accuracy.

The set-up of a GEM model application for a specific area and period comprises, besides the GEM set of processes and parameter setting, input for schematisation and transport, loadings, boundaries, forcings and initial conditions. GEM can be combined with any hydrodynamic model and additional processes to create a dedicated model application for a specific ecosystem.

\section{Model set-up}

GEM comprises a set of process formulations quantifying the $\mathrm{P}$ term of the advection-diffusion equation. The set of process formulations is dedicated to modelling the nutrient cycling and primary production in coastal and estuarine systems. The approach for the development of GEM started from an existing ecological model at WL I Delft Hydraulics, which proved useful for the simulation of eutrophication in Dutch coastal waters (e.g. Los \& Bokhorst, 1997; De Vries et al., 1998). In a series of 
research and consultancy projects, partly in cooperation with other Dutch institutes for marine research, GEM has been further elaborated and improved. The resulting GEM includes default parameter settings that have been calibrated for the North Sea and that have proven to be applicable for a range of other coastal ecosystems as well.

GEM simulates the nutrient cycles of nitrogen $(\mathrm{N})$, phosphorus $(\mathrm{P})$ and silicate $(\mathrm{Si})$. For the dissolved inorganic state of these nutrients, the following state variables are included in the model: nitrate $\left(\mathrm{NO}_{3}\right)$ representing the sum of nitrite and nitrate, ammonia $\left(\mathrm{NH}_{4}\right)$, ortho-phosphate $\left(\mathrm{PO}_{4}\right)$ and dissolved silicate (Si). Four phytoplankton species groups are simulated: diatoms, flagellates, dinoflagellates and Phaeocystis. Dead particulate organic matter in water is included as separate variables for particulate organic carbon (POC), particulate organic nitrogen (PON), particulate organic phosphorus (POP) and opal silicate (POSi). Similarly, four organic matter variables are defined in the sediment $\left(\mathrm{POC}_{\mathrm{S}}, \mathrm{PON}_{\mathrm{S}}\right.$, $\mathrm{POP}_{\mathrm{S}}$ and $\mathrm{POSi}_{\mathrm{S}}$ ). Additional model variables are dissolved oxygen $\left(\mathrm{O}_{2}\right)$ and salinity (SAL). Suspended matter concentrations and water temperature are forcing parameters in the model.

\section{Processes included in GEM}

Figure 1 gives a schematic overview of the variables and processes incorporated in the present set-up of GEM. GEM includes the following processes:

- phytoplankton processes: primary production, respiration and mortality

- extinction of light

- decomposition of particulate organic matter in water and sediment

- nitrification and denitrification

- reaeration

- settling

- burial

- filterfeeder processes: grazing, excretion and respiration

The above-mentioned processes in GEM are described in more detail below. For some processes, alternative (more detailed) formulations are available and additional processes are available as well. These are used for specific model applications that require more detailed process formulations, for example, for nutrients in the sediment, phosphate adsorption, salinity effects on mortality and decomposition of organic matter or benthic algae. In this paper, however, we focus on the set of formulations that is most commonly used. Equations used in GEM, state variables and process parameter (default) settings, and phytoplankton parameter values in the BLOOM module are presented in Tables $1-3$, respectively.

\section{Phytoplankton processes}

In GEM, the phytoplankton module, BLOOM, simulates primary production, respiration and mortality of phytoplankton. This module allows for the modelling of species competition and adaptation of phytoplankton to limiting nutrients or light (Los et al., 1984; Los \& Brinkman, 1988; Van der Molen et al., 1994; Los \& Bokhorst, 1997; Los, 2005). For the simulation of species competition, four species groups are defined in GEM: diatoms, flagellates, dinoflagellates and Phaeocystis. BLOOM can also be used for other species groups, including fresh water species and benthic species, but these are generally not used in GEM. Within each of these groups, three phenotypes are defined to account for adaptation to changing environmental conditions:

- energy types, with relatively high growth rates, low mortality rates and high N/C and $\mathrm{P} / \mathrm{C}$ ratio

- nitrogen types, with typically lower internal N/C ratio, lower maximum growth rates, higher mortality rates, higher settling velocities and higher chlorophyll content

- phosphorus types, with typically lower internal P/ $\mathrm{C}$ ratio, lower maximum growth rates, higher mortality rates, lower settling velocities and lower chlorophyll-a content

The different phenotypes of a species group are modelled as separate variables with different parameter settings, e.g. growth rates, settling velocities and respiration rates. When conditions in the water change, biomass of one phenotype can be instantaneously converted into another phenotype of the same species group, representing rapid adaptation of individual algal cells. Since the phytoplankton types represent different phenotypes of the same species, the transition of one type to another occurs at the time scale of a cell division, which is in the order of a day. Due to this characteristic time scale (i.e. Chapter 5 of 


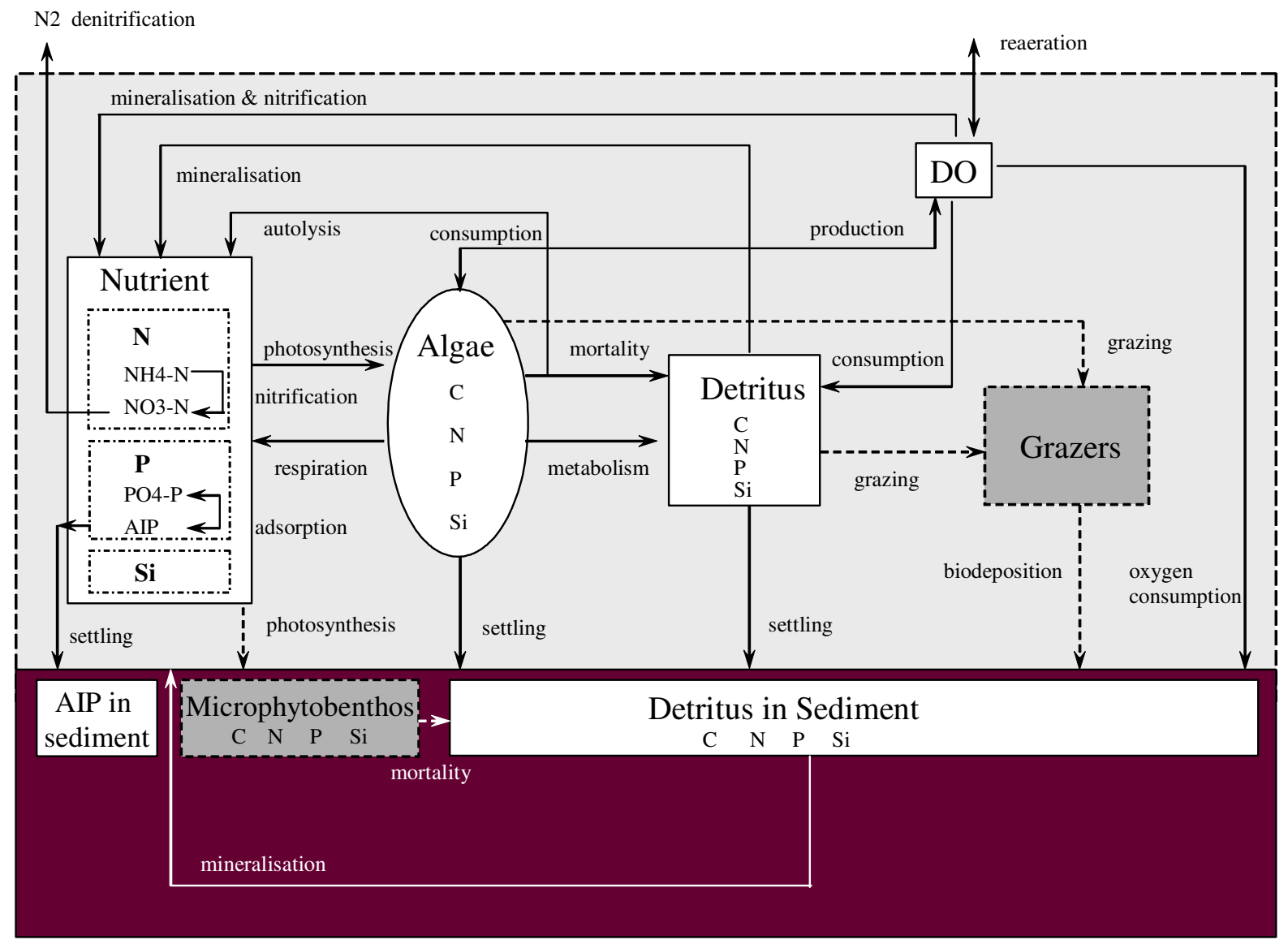

Fig. 1 Schematic overview of all state variables and processes included in the ecological model instrument GEM. State variables in grey and processes indicated by dashed lines are

Harris, 1986), the simulation time step for the BLOOM phytoplankton processes is usually chosen to be $24 \mathrm{~h}$. Nutrient fluxes associated with switches between types are dealt with in the following way: If the nutrient content requires a larger amount of nutrients than previously stored in the phytoplankton, it is taken up from the dissolved fraction in the water. If, in contrast, the nutrient content declines, the extraneous amount previously stored in the phytoplankton is released into the dissolved fraction in the water. This is similar to autolysis. Switch-associated uptake of one nutrient and release of another may occur simultaneously, i.e. when a P-type is replaced by an N-type. A shift in species composition due to changing environmental conditions is a slower process, involving changing dominance of species groups. This shift in species composition is restricted by growth and mortality rates. BLOOM allows for optional andhave not been included in the North Sea modelling applications. AIP is 'adsorbed inorganicphosphorus'

simultaneous co-existence of different phenotypes and/or species groups, representing a continuous variable stoichiometry restricted only by the limits set for the individual types. Notice that the stoichiometry of the types is fixed, but the relative proportion varies (see Los et al. (2008) for a more detailed description of BLOOM). BLOOM was originally developed as a steady-state model that is now being applied in a dynamic setting. In a typical simulation, transport and a small number of processes are simulated with a short time step (i.e. $30 \mathrm{~min}$ of less), while the majority of processes are simulated using a much longer time step of $24 \mathrm{~h}$. Consistency and mass conservation issues due to the different time steps and approaches are resolved by the numerical solver of the mode. Comparative simulations have shown that the results of simulations with a $30 \mathrm{~min}$ time step for all processes are similar to those with 
Table 1 Equations in GEM

A. Balance equations for state variables:

$$
\begin{aligned}
& \frac{d N O_{3}}{d t}=n i t-d e n-u p t_{N} *\left(1-f_{a m}\right) \\
& \frac{d N H_{4}}{d t}=\operatorname{dec}_{P O N}-n i t+\operatorname{dec}_{P O N_{S}}+r s p_{N, G}+f_{\text {aut }} * \operatorname{mor}_{N}-u p t_{N} * f_{a m} \\
& \frac{d P O_{4}}{d t}=\operatorname{dec}_{P O P}+\operatorname{dec}_{P O P_{S}}+\operatorname{rsp}_{P, G}+f_{\text {aut }} * \operatorname{mor}_{P}-u p t_{P} \\
& \frac{d S i}{d t}=\operatorname{dec}_{P O S i}+\operatorname{dec}_{P_{O S i_{S}}}+f_{\text {aut }} * \text { mor }_{S i}-u_{\mathrm{P} t_{S i}} \\
& \frac{d A L G_{i}}{d t}=g r o_{i}-m_{r} t_{i}-\operatorname{sed}_{i}-g r z_{i} \\
& \frac{d O_{2}}{d t}=r e a+\left(g r_{C}-\operatorname{dec}_{P O C}-\operatorname{dec}_{P O C_{S}}\right) * s_{o}-\text { nit } * s_{n o} \\
& \frac{d P O X}{d t}=\operatorname{mor}_{X} *\left(1-f_{\text {aut }}\right)-\operatorname{sed}_{P O X}-\operatorname{dec}_{P O X}-\operatorname{grz}_{P O X}+\operatorname{exc}_{P O X} \\
& \frac{d P O X_{S}}{d t}=\left(\operatorname{sed}_{P O X}-\operatorname{dec}_{P O X_{S}}-\operatorname{bur}_{P O X_{S}}+\operatorname{exc}_{P O X_{S}}\right) * Z
\end{aligned}
$$

where:

$i=$ algae type $1-12$

$\mathrm{X}=$ element carbon, nitrogen, phosphorus and silicate

$$
\begin{aligned}
& \text { upt }_{X}=\sum_{i=1}^{n}\left(\text { gro }_{i} * s_{X, i}\right) \\
& \text { mor }_{X}=\sum_{i=1}^{n}\left(\text { mrt }_{i} * s_{X, i}\right) \\
& f_{a m}=\frac{\operatorname{MIN}\left(\mathrm{NH}_{4}, \mathrm{upt}_{\mathrm{N}} * \Delta \mathrm{t}\right)}{u p t_{N} * \Delta t}
\end{aligned}
$$

B. Phytoplankton processes

$$
\begin{aligned}
& \text { gro }_{i}=\frac{A L G_{i, n e w}-A L G_{i}}{\Delta t}+m r t_{i} \\
& m r t_{i}=m_{i} * A L G_{i} \\
& p_{i}=p_{i, 0} *\left(T-k t_{p, i}\right) \\
& r_{i}=r_{i, 0} *\left(k t_{r, i}\right)^{T} \\
& m_{i}=m_{i, 0} *\left(k t_{m, i}\right)^{T}
\end{aligned}
$$

Optimisation: find a set of new concentrations of $\mathrm{ALG}_{i}$ with maximum:

$\sum_{i=1}^{n}\left(p g_{i} * l e_{i}-r_{i}\right) * A L G_{i}$

$\sum_{i=1}^{3} A L G_{i, \text { new }} \leq \sum_{i=1}^{3}\left(A L G_{i}\right) \mathrm{e}^{\left(p g_{i} * l e_{i}-r_{i}\right) * \Delta t}$

(growth constraint per species group) (B.8)

$\sum_{i=1}^{3} A L G_{i, n e w} \geq \sum_{i=1}^{3} A L G_{i} * \mathrm{e}^{-m_{i} * \Delta t}$

(mortality constraint per species group) (B.9)

$\sum_{i=1}^{n}\left(s_{N, i} * A L G_{i_{\text {new }}}\right) \leq \sum_{i=1}^{n}\left(s_{N, i} * A L G_{i}\right)+\mathrm{NO}_{3}+\mathrm{NH}_{4}$

(nutrient constraint, nitrogen) (B.10)

$\sum_{i=1}^{n}\left(s_{P, i} * A L G_{i, \text { new }}\right) \leq \sum_{i=1}^{n}\left(s_{P, i} * A L G_{i}\right)+P O_{4}$

(nutrient constraint, phosphorus) (B.11)

$\sum_{i=1}^{n}\left(s_{S i, i} * A L G_{i, \text { new }}\right) \leq \sum_{i=1}^{n}\left(s_{S i, i} * A L G_{i}\right)+S i$

(nutrient constraint, silicate) (B.12)

$k_{\text {min }, i} \leq k_{d} \leq k_{\text {max }, i}$

(light constraint) (B.13)

where:

$k_{\max , i}=f_{\text {table }}\left(l e_{c r}\right)$

$l e_{c r}=\frac{m_{i}+r_{i}}{p g_{i}}$

C. Extinction of light

$k_{d}=k_{b}+k_{S P M}+k_{P O M}+k_{A L G}+k_{H U M}$

$k_{P O M}=e_{P O C} * P O C$

$k_{A L G}=\sum_{i=1}^{n}\left(e_{A L G_{i}} * A L G_{i}\right)$

$k_{S P M}=e_{S P M} * S P M$

$k_{H U M}=e_{H U M, 0} *\left(1-\frac{S A L}{S A L_{b}}\right)$ 
Table 1 continued

D. Decomposition of organic matter

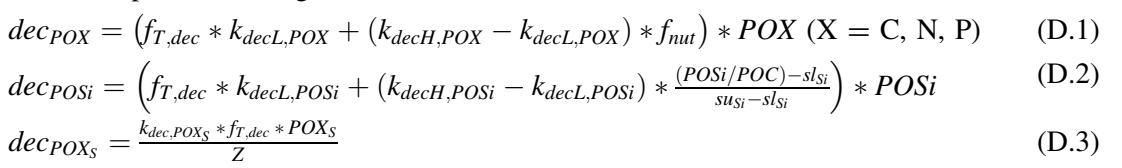

where:

$f_{T, p r}=k r_{p r}^{(T-20)}$ where: $\mathrm{pr}=\mathrm{dec}$, den, nit

$f_{n u t}=\operatorname{MIN}\left(\frac{P O N / P O C)-s l_{N}}{s u_{N}-s l_{N}}, \frac{P O P / P O C)-s l_{P}}{s u_{P}-s l_{P}}\right)$

E. Nitrification and denitrification

nit $=k_{\text {nit }} * \mathrm{NH}_{4} * f_{T, \text { nit }}$

den $=k_{\text {den }} * \mathrm{NO}_{3} * f_{T, \text { den }}$

F. Reaeration

rea $=\left(0.3+0.028 * W^{2}\right) * \frac{\left(O_{2, e q}-O_{2}\right)}{Z} * k_{\text {rea }}$

G. Sedimentation

$\operatorname{sed}_{Y}=\frac{v_{Y} * Y}{Z}$ where: $\mathrm{Y}=\mathrm{ALG}_{i}, \mathrm{POX}$

H. Burial

bur $_{P O X_{S}}=\frac{b * P O X_{S}}{Z}$

I. Filterfeeder processes

$g r z_{i}=u p * A L G_{i}$

grz $_{P O X}=u p * P O X$

$r s p_{X, G}=\left(\right.$ ass $\left.2 * r g_{G, 20}+G_{i} * r m_{G, 20}\right) * f_{T, g r z} * s_{X, G}$

$\operatorname{exc}_{P O X}=\operatorname{exc}_{X} *\left(1-f_{\text {sed }}\right)$

$\operatorname{exc}_{P O X_{S}}=\operatorname{exc}_{X} * f_{\text {sed }}$

where:

$e x c_{X}=e g_{X}+a s s 1_{X}-\frac{a s s 4 * s_{X, G}}{\Delta t}-a s s 2 * r g_{G, 20} * f_{T, g r z} * s_{X, G}$

up $=G * f_{T, g r z} * \operatorname{MIN}\left(k_{f l t, 20} \frac{c_{f}}{c_{f}+k_{f}}, k_{\text {upt }, 20} \cdot \frac{1}{c_{f}}\right)$

$c_{f}=\sum_{i=1}^{n}\left(A L G_{i}\right)+P O C$

$e g_{X}=g r z_{P O X} * f_{e g}+\sum_{i=1}^{n}\left(g r z_{i} * f_{e g} * s_{X, i}\right)$

$\operatorname{ass}_{X}=g r z_{P O X}+\sum_{i=1}^{n}\left(g r z_{i} * s_{X, i}\right)-e g_{X}$

ass $2=\operatorname{MIN}\left(\operatorname{ass}_{C}, \frac{a s s 1_{N}}{s_{N, G}}, \frac{a s s 1_{P}}{s_{P, G}}\right)$

$\operatorname{ass} 3=\operatorname{ass} 2 *\left(1-r g_{G, 20} * e^{k t_{G} *(T-20)}\right)$

ass $4=\operatorname{MIN}\left(\right.$ ass $\left.3 * \Delta t, G_{c}-G *\left(1-r m_{G, 20} * f_{T, g r z} * \Delta t\right)\right)$

$G_{c}=\operatorname{MAX}\left(G_{i}, G *\left(1+g_{G, 20} * f_{T, g r z} * \Delta t\right)\right.$ If $\mathrm{G}_{\mathrm{i}}>\mathrm{G}$

$G_{c}=\operatorname{MIN}\left(G_{i}, G *\left(1-m_{G, 20} * f_{T, g r z} * \Delta t\right)\right.$ If $\mathrm{G}_{\mathrm{i}}<\mathrm{G}$

$G_{\text {new }}=\operatorname{MIN}\left(G_{c}, G *\left(1-r m_{G, 20} * f_{T, \text { grz }} * \Delta t\right)+\operatorname{ass} 3 * \Delta t\right.$

$f_{T, g r z}=e^{k t_{G}(T-20)}$

variable time steps (see Los et al., 2008 for further details).

BLOOM assumes that fast-growing (less efficient) phytoplankton species dominate in a situation where resources (light, nutrients) are abundant, while slowgrowing but efficient phytoplankton species gain dominance when resources become limited. This assumption is based on the theory on $\mathrm{k}$ - and r-strategies (e.g. Reynolds et al., 1983; Harris, 1986). Linear programming is used as an optimisation technique to determine the species composition that is best adapted to prevailing environmental 
Table 2 Explanation of symbols

\begin{tabular}{|c|c|c|c|}
\hline Symbol & Description & Value & Unit \\
\hline & State variables & & \\
\hline $\mathrm{NO}_{3}$ & Nitrate & & $\mathrm{gN} \mathrm{m}^{-3}$ \\
\hline $\mathrm{NH}_{4}$ & Ammonium & & $\mathrm{gN} \mathrm{m}^{-3}$ \\
\hline $\mathrm{PO}_{4}$ & Ortho-phosphate & & $\mathrm{gP} \mathrm{m}^{-3}$ \\
\hline $\mathrm{Si}$ & Dissolved silicate & & $\mathrm{gSi} \mathrm{m}^{-3}$ \\
\hline $\mathrm{O}_{2}$ & Dissolved oxygen & & $\mathrm{gO}_{2} \mathrm{~m}^{-3}$ \\
\hline $\mathrm{ALG}_{1}$ & Diatoms energy type: diat-E & & $\mathrm{gC} \mathrm{m}^{-3}$ \\
\hline $\mathrm{ALG}_{2}$ & Diatoms nitrogen type: diat-N & & $\mathrm{gC} \mathrm{m}^{-3}$ \\
\hline $\mathrm{ALG}_{3}$ & Diatoms phosphorus type: diat-P & & $\mathrm{gC} \mathrm{m}^{-3}$ \\
\hline $\mathrm{ALG}_{4}$ & Flagellates energy type: flag-E & & $\mathrm{gC} \mathrm{m}^{-3}$ \\
\hline $\mathrm{ALG}_{5}$ & Flagellates nitrogen type: flag-N & & $\mathrm{gC} \mathrm{m}^{-3}$ \\
\hline $\mathrm{ALG}_{6}$ & Flagellates phosphorus type: flag-P & & $\mathrm{gC} \mathrm{m}^{-3}$ \\
\hline $\mathrm{ALG}_{7}$ & Dinoflagellates energy type: dino-E & & $\mathrm{gC} \mathrm{m}^{-3}$ \\
\hline $\mathrm{ALG}_{8}$ & Dinoflagellates nitrogen type: dino-N & & $\mathrm{gC} \mathrm{m} \mathrm{m}^{-3}$ \\
\hline $\mathrm{ALG}_{9}$ & Dinoflagellates phosphorus type: dino-P & & $\mathrm{gC} \mathrm{m}^{-3}$ \\
\hline $\mathrm{ALG}_{10}$ & Phaeocystis energy type: Phaeo-E & & $\mathrm{gC} \mathrm{m}^{-3}$ \\
\hline $\mathrm{ALG}_{11}$ & Phaeocystis nitrogen type: Phaeo-N & & $\mathrm{gC} \mathrm{m}^{-3}$ \\
\hline $\mathrm{ALG}_{12}$ & Phaeocystis phosphorus type: Phaeo-P & & $\mathrm{gC} \mathrm{m}{ }^{-3}$ \\
\hline POC & Particulate organic carbon & & $\mathrm{gC} \mathrm{m}^{-3}$ \\
\hline PON & Particulate organic nitrogen & & $\mathrm{gN} \mathrm{m}^{-3}$ \\
\hline POP & Particulate organic phosphorus & & $\mathrm{gP} \mathrm{m}^{-3}$ \\
\hline POSi & Particulate organic silicate & & $\mathrm{gSi} \mathrm{m}^{-3}$ \\
\hline $\mathrm{POC}_{\mathrm{S}}$ & Particulate organic carbon in the sediment & & $\mathrm{gC} \mathrm{m}^{-2}$ \\
\hline $\mathrm{PON}_{S}$ & Particulate organic nitrogen in the sediment & & $\mathrm{gN} \mathrm{m}^{-2}$ \\
\hline $\mathrm{POP}_{\mathrm{S}}$ & Particulate organic phosphorus in the sediment & & $\mathrm{gP} \mathrm{m}^{-2}$ \\
\hline $\mathrm{POSi}_{\mathrm{S}}$ & Particulate organic silicate in the sediment & & $\mathrm{gSi} \mathrm{m}^{-2}$ \\
\hline \multirow[t]{2}{*}{ SAL } & Salinity & & ppt \\
\hline & Fluxes & & \\
\hline sed & Settling & & $\mathrm{gX} \mathrm{m} \mathrm{m}^{-3} \mathrm{~d}^{-1}$ \\
\hline mrt & Phytoplankton mortality & & $\mathrm{gC} \mathrm{m}^{-3} \mathrm{~d}^{-1}$ \\
\hline gro & Net phytoplankton growth & & $\mathrm{gC} \mathrm{m}^{-3} \mathrm{~d}^{-1}$ \\
\hline $\operatorname{mor}_{\mathrm{X}}$ & Formation of dead organic matter by phytoplankton mortality & & $\mathrm{gX} \mathrm{m}^{-3} \mathrm{~d}^{-1}$ \\
\hline upt $_{\mathrm{X}}$ & Uptake of nutrients by phytoplankton growth & & $\mathrm{gX} \mathrm{m}^{-3} \mathrm{~d}^{-1}$ \\
\hline $\operatorname{dec}_{X}$ & Decomposition of dead particulate organic matter & & $\mathrm{gX} \mathrm{m} \mathrm{m}^{-3} \mathrm{~d}^{-1}$ \\
\hline $\operatorname{bur}_{X}$ & Burial & & $\mathrm{gX} \mathrm{m} \mathrm{m}^{-3} \mathrm{~d}^{-1}$ \\
\hline den & Denitrification & & $\mathrm{gN} \mathrm{m}^{-3} \mathrm{~d}^{-1}$ \\
\hline nit & Nitrification & & $\mathrm{gN} \mathrm{m}^{-3} \mathrm{~d}^{-1}$ \\
\hline rea & Reaeration & & $\mathrm{gO}_{2} \mathrm{~m}^{-3} \mathrm{~d}^{-1}$ \\
\hline $\operatorname{grz}_{\mathrm{X}}$ & Grazing by filterfeeders & & $\mathrm{gX} \mathrm{m}^{-3} \mathrm{~d}^{-1}$ \\
\hline $\operatorname{rsp}_{\mathrm{X}, \mathrm{G}}$ & Respiration by filterfeeders & & $\mathrm{gX} \mathrm{m}^{-3} \mathrm{~d}^{-1}$ \\
\hline \multirow[t]{2}{*}{$\operatorname{exc}_{X}$} & Excretion of organic matter by filterfeeders & & $\mathrm{gX} \mathrm{m}^{-3} \mathrm{~d}^{-1}$ \\
\hline & Explanation of symbols & & \\
\hline$\Delta t$ & Time step for processes (different from that of transport) & 1 & d \\
\hline $\mathrm{ALG}_{i, \text { new }}$ & Concentration of algae type $i$ at the end of the time step & $* *$ & $\mathrm{gC} \mathrm{m}^{-3}$ \\
\hline $\operatorname{ass} 1_{\mathrm{X}}$ & Net uptake flux of food of nutrient $\mathrm{X}(\mathrm{C}, \mathrm{N}$ or $\mathrm{P})$ & $* *$ & $\mathrm{gX} \mathrm{m} \mathrm{m}^{-3} \mathrm{~d}^{-1}$ \\
\hline
\end{tabular}


Table 2 continued

\begin{tabular}{|c|c|c|c|}
\hline Symbol & Description & Value & Unit \\
\hline ass 2 & Net uptake flux corrected for stoichiometry filterfeeders & $* *$ & $\mathrm{gC} \mathrm{m}^{-3} \mathrm{~d}^{-1}$ \\
\hline ass 3 & Maximum assimilation flux corrected for growth respiration & $* *$ & $\mathrm{gC} \mathrm{m}^{-3} \mathrm{~d}^{-1}$ \\
\hline ass 4 & Effectively assimilated organic matter during time step & $* *$ & $\mathrm{gC} \mathrm{m}^{-3}$ \\
\hline$b$ & Burial rate & 0.0025 & $\mathrm{~d}^{-1}$ \\
\hline$c_{\mathrm{f}}$ & Available food concentration & $* *$ & $\mathrm{gC} \mathrm{m}^{-3}$ \\
\hline $\mathrm{Cl}$ & Chloride concentration & $* * *$ & $\mathrm{~g} \mathrm{~m}^{-3}$ \\
\hline$e_{\mathrm{ALG} i}$ & Specific extinction of algae type $i$ & $*$ & $\mathrm{~m}^{2} \mathrm{~g} \mathrm{C}^{-1}$ \\
\hline $\operatorname{eg}_{\mathrm{X}}$ & Egestion flux by grazers of nutrient $\mathrm{X}(\mathrm{C}, \mathrm{N}$ or $\mathrm{P})$ & $* *$ & $\mathrm{gX} \mathrm{m}^{-3} \mathrm{~d}^{-1}$ \\
\hline$e_{\mathrm{HUM}, 0}$ & Extinction due to humic substances in pure freshwater & 0.97 & $\mathrm{~m}^{-1}$ \\
\hline$e_{\mathrm{POC}}$ & Specific extinction of particulate dead organic matter & 0.1 & $\mathrm{~m}^{2} \mathrm{~g} \mathrm{C}^{-1}$ \\
\hline$e_{\mathrm{SPM}}$ & Specific extinction of inorganic suspended matter & 0.025 & $\mathrm{~m}^{2} \mathrm{~g}^{-1}$ \\
\hline$f_{\mathrm{am}}$ & Fraction of ammonium in nitrogen uptake & $* *$ & - \\
\hline$f_{\text {aut }}$ & Autolysis fraction of mortality & 0.3 & - \\
\hline$f_{\mathrm{eg}}$ & Fraction of uptake that is egested by grazers & 0.5 & - \\
\hline$f_{\text {nut }}$ & Function for relative nutrient availability & $* *$ & - \\
\hline$f_{\text {sed }}$ & Fraction of excretion by grazers released to sediment & 1 & - \\
\hline$f_{\text {table }}$ & $\begin{array}{l}\text { Tabulated function relating } \mathrm{k}_{\mathrm{d}} \text { to growth efficiency, converted from the } \\
\text { tabulated function of growth efficiency and light }\end{array}$ & $* *$ & $\mathrm{~m}^{-1}$ \\
\hline$f_{\mathrm{T}, \mathrm{pr}}$ & Temperature function for process $\mathrm{pr}=\mathrm{dec}, \mathrm{den}$, nit & $* *$ & - \\
\hline$f_{\mathrm{T}, \mathrm{grz}}$ & Temperature function for grazing processes & $* *$ & - \\
\hline G & Grazer biomass & $* *$ & $\mathrm{gC} \mathrm{m}^{-3}$ \\
\hline$G_{\mathrm{c}}$ & Feasible grazer biomass at maximum growth or mortality rates & $* *$ & $\mathrm{gC} \mathrm{m}^{-3}$ \\
\hline$g_{\mathrm{G}, 20}$ & Maximum growth rate grazers at $20^{\circ} \mathrm{C}$ & 0.2 & $\mathrm{~d}^{-1}$ \\
\hline$G_{\mathrm{i}}$ & Imposed grazer biomass by forcing function & $* * *$ & $\mathrm{gC} \mathrm{m}^{-3}$ \\
\hline$G_{\text {new }}$ & Effective grazer biomass at the end of the time step & $* *$ & $\mathrm{gC} \mathrm{m}^{-3}$ \\
\hline$k_{\mathrm{ALG}}$ & Total extinction due to phytoplankton & $* *$ & $\mathrm{~m}^{-1}$ \\
\hline$k_{\mathrm{b}}$ & Background extinction & 0.08 & $m^{-1}$ \\
\hline$k_{\mathrm{d}}$ & Total extinction coefficient & $* *$ & $\mathrm{~m}^{-1}$ \\
\hline$k_{\mathrm{decL}, \mathrm{POC}}$ & Minimum decomposition rate for POC at $20^{\circ} \mathrm{C}$ & 0.12 & $d^{-1}$ \\
\hline$k_{\mathrm{decL}, \mathrm{PON}}$ & Minimum decomposition rate for $\mathrm{PON}$ at $20^{\circ} \mathrm{C}$ & 0.08 & $d^{-1}$ \\
\hline$k_{\mathrm{decL}, \mathrm{POP}}$ & Minimum decomposition rate for POP at $20^{\circ} \mathrm{C}$ & 0.08 & $\mathrm{~d}^{-1}$ \\
\hline$k_{\mathrm{decL}, \mathrm{POSi}}$ & Minimum decomposition rate for POSi at $20^{\circ} \mathrm{C}$ & 0.04 & $d^{-1}$ \\
\hline$k_{\mathrm{dec}, \mathrm{POCS}}$ & Decomposition rate for $\mathrm{POC}$ in sediment at $20^{\circ} \mathrm{C}$ & 0.015 & $d^{-1}$ \\
\hline$k_{\mathrm{dec}, \mathrm{PONS}}$ & Decomposition rate for $\mathrm{PON}$ in sediment at $20^{\circ} \mathrm{C}$ & 0.015 & $\mathrm{~d}^{-1}$ \\
\hline$k_{\mathrm{dec}, \mathrm{POPS}}$ & Decomposition rate for POP in sediment at $20^{\circ} \mathrm{C}$ & 0.025 & $d^{-1}$ \\
\hline$k_{\mathrm{dec}, \mathrm{POSiS}}$ & Decomposition rate for POSi in sediment at $20^{\circ} \mathrm{C}$ & 0.008 & $d^{-1}$ \\
\hline$k_{\mathrm{decH}, \mathrm{POC}}$ & Maximum decomposition rate for $\mathrm{POC}$ at $20^{\circ} \mathrm{C}$ & 0.18 & $d^{-1}$ \\
\hline$k_{\mathrm{decH}, \mathrm{PON}}$ & Maximum decomposition rate for PON at $20^{\circ} \mathrm{C}$ & 0.18 & $\mathrm{~d}^{-1}$ \\
\hline$k_{\mathrm{decH}, \mathrm{POP}}$ & Maximum decomposition rate for POP at $20^{\circ} \mathrm{C}$ & 0.18 & $d^{-1}$ \\
\hline$k_{\mathrm{decH}, \mathrm{POSi}}$ & Maximum decomposition rate for POSi at $20^{\circ} \mathrm{C}$ & 0.08 & $d^{-1}$ \\
\hline$k_{\text {den }}$ & Denitrification rate & 0.003 & $d^{-1}$ \\
\hline$k_{\mathrm{f}}$ & Half saturation constant for grazing & 0.1 & $\mathrm{gC} \mathrm{m}^{-3}$ \\
\hline$k_{\mathrm{flt}, 20}$ & Maximum rate of filtration by mussels & 0.05 & $\mathrm{~m}^{3} \mathrm{gC}^{-1} \mathrm{~d}^{-1}$ \\
\hline$k_{\mathrm{HUM}}$ & Extinction due to humic substances from freshwater input & $* *$ & $\mathrm{~m}^{-1}$ \\
\hline
\end{tabular}


Table 2 continued

\begin{tabular}{|c|c|c|c|}
\hline Symbol & Description & Value & Unit \\
\hline$k_{\max , I}$ & $\begin{array}{l}\text { Maximum extinction where the net growth of algae type } i \text { is positive; } \\
\text { above this level, self-shading limits growth }\end{array}$ & $* *$ & $\mathrm{~m}^{-1}$ \\
\hline$k_{\min , I}$ & $\begin{array}{l}\text { Minimum extinction where the net growth of algae type } i \text { is positive; } \\
\text { below this level, photo-inhibition limits growth }\end{array}$ & 0 & $\mathrm{~m}^{-1}$ \\
\hline$k_{\text {nit }}$ & Nitrification rate & 0.07 & $\mathrm{~d}^{-1}$ \\
\hline$k_{\mathrm{POM}}$ & Extinction of dead particulate organic matter & $* *$ & $\mathrm{~m}^{2} \mathrm{gC}^{-1}$ \\
\hline$k_{\text {rea }}$ & Reaeration rate & 4 & $\mathrm{~d}^{-1}$ \\
\hline$k_{\text {SPM }}$ & Extinction of inorganic suspended matter & $* *$ & $\mathrm{~m}^{2} \mathrm{gC}^{-1}$ \\
\hline$k t_{\mathrm{dec}}$ & $\begin{array}{l}\text { Temperature coefficient for decomposition of POX and } \mathrm{POX}_{\mathrm{S}} \text {, except for opal } \\
\text { silicate in sediment }\end{array}$ & 1.11 & - \\
\hline$k t_{\mathrm{dec}, \mathrm{POSiS}}$ & Temperature coefficient for dissolution of opal silicate in sediment & 1.047 & - \\
\hline$k t_{\mathrm{den}}$ & Temperature coefficient for denitrification & 1.11 & - \\
\hline$k t_{\mathrm{G}}$ & Temperature coefficient for grazing processes & 0.04 & - \\
\hline$k t_{\mathrm{m}, i}$ & Temperature coefficient for mortality of algae type $i$ & $*$ & - \\
\hline$k t_{\text {nit }}$ & Temperature coefficient for nitrification & 1.06 & - \\
\hline$k t_{\mathrm{p}, i}$ & Temperature coefficient for phytoplankton growth & * & ${ }^{\circ} \mathrm{C}$ \\
\hline$k t_{\mathrm{r}, i}$ & Temperature coefficient for phytoplankton respiration & 1.07 & - \\
\hline$k_{\mathrm{upt}, 20}$ & Maximum food uptake rate by grazers & 0.1 & $\mathrm{gC} \mathrm{gC}^{-1} \mathrm{~d}^{-1}$ \\
\hline$l e_{\mathrm{cr}}$ & Critical light efficiency where phytoplankton growth just balances losses & $* *$ & - \\
\hline$l e_{\mathrm{i}}$ & Growth efficiency of algae type $i$, tabulated function of light & $* *$ & - \\
\hline$m_{i, 0}$ & Mortality rate for algae type $i$ at $0^{\circ} \mathrm{C}$ & * & $d^{-1}$ \\
\hline$m_{\mathrm{G}, 20}$ & Maximum mortality rate of grazers at $20^{\circ} \mathrm{C}$ & 0.2 & $d^{-1}$ \\
\hline$m_{i}$ & Mortality rate for algae type $i$ & $* *$ & $d^{-1}$ \\
\hline$n$ & Number of algae types in calculation & 12 & - \\
\hline $\mathrm{O}_{2, \text { eq }}$ & Saturation concentration of oxygen & $* *$ & $\mathrm{gO}_{2} \mathrm{~m}^{-3}$ \\
\hline$p_{i, 0}$ & Maximal net growth rate of algae type $i$ at $0^{\circ} \mathrm{C}$ & $*$ & $\mathrm{~d}^{-1}$ \\
\hline$p g_{i}$ & Maximal gross growth rate algae type $i$ & $* *$ & $d^{-1}$ \\
\hline$p_{i}$ & Maximal net growth rate for algae type $i$ & $* *$ & $\mathrm{~d}^{-1}$ \\
\hline$r_{i, 0}$ & Maintenance respiration rate for algae type $i$ at $0^{\circ} \mathrm{C}$ & 0.06 & $\mathrm{~d}^{-1}$ \\
\hline$r g_{\mathrm{G}, 20}$ & Growth respiration fraction of grazers at $20^{\circ} \mathrm{C}$ & 0.2 & - \\
\hline$r m_{\mathrm{G}, 20}$ & Maintenance respiration rate of grazers at $20^{\circ} \mathrm{C}$ & 0.005 & $d^{-1}$ \\
\hline$r_{i}$ & Maintenance respiration rate for algae type $i$ & $* *$ & $\mathrm{~d}^{-1}$ \\
\hline $\mathrm{SAL}_{\mathrm{b}}$ & Background salinity & 34.97 & ppt \\
\hline$s l_{\mathrm{N}}$ & Lower limit stoichiometric constant PON & 0.1 & $\mathrm{Gn} \mathrm{gC}^{-1}$ \\
\hline$s l_{\mathrm{P}}$ & Lower limit stoichiometric constant POP & 0.01 & $\mathrm{gP} \mathrm{gC}^{-1}$ \\
\hline$s l_{\mathrm{Si}}$ & Lower limit stoichiometric constant POSi & 0.01 & $\mathrm{gSi}_{\mathrm{gC}} \mathrm{gC}^{-1}$ \\
\hline$s_{\mathrm{N}, G}$ & Stochiometry of nutrient $\mathrm{N}$ in grazers & 0.1818 & $\mathrm{gN} \mathrm{gC}^{-1}$ \\
\hline$s_{\mathrm{NO}}$ & Oxygen nitrogen ratio in $\mathrm{NO}_{3}$ & 4.571 & $\mathrm{gO}_{2} \mathrm{gN}^{-1}$ \\
\hline$s_{\mathrm{O}}$ & Oxygen carbon ratio in detritus & 2.67 & $\mathrm{gO}_{2} \mathrm{gC}^{-1}$ \\
\hline$s_{\mathrm{P}, G}$ & Stoichiometry of nutrient $\mathrm{P}$ in grazers & 0.0263 & $\mathrm{gP} \mathrm{gC}^{-1}$ \\
\hline SPM & (Suspended) inorganic matter concentration & $* * *$ & $\mathrm{gDM} \mathrm{m}^{-3}$ \\
\hline$s u_{\mathrm{N}}$ & Upper limit stoichiometric constant PON & 0.15 & $\mathrm{gN} \mathrm{gC}^{-1}$ \\
\hline$s u_{\mathrm{P}}$ & Upper limit stoichiometric constant POP & 0.015 & $\mathrm{gP} \mathrm{gC}^{-1}$ \\
\hline$s u_{\mathrm{Si}}$ & Upper limit stoichiometric constant POSi & 0.005 & $\mathrm{gSi}_{\mathrm{gC}} \mathrm{g}^{-1}$ \\
\hline$s_{X, i}$ & Stoichiometry of nutrient $\mathrm{X}$ in algae type $i$ & $*$ & $\mathrm{gX} \mathrm{gC}^{-1}$ \\
\hline
\end{tabular}


Table 2 continued

\begin{tabular}{llll}
\hline Symbol & Description & Value & Unit \\
\hline$T$ & Water temperature & $* * *$ & ${ }^{\circ} \mathrm{C}$ \\
$u p$ & Uptake rate of organic matter by grazers & $* *$ & $\mathrm{~d}^{-1}$ \\
$v_{\mathrm{ALG} i}$ & Settling velocity of algae type $i$ & $*$ & $\mathrm{~m} \mathrm{~d}^{-1}$ \\
$v_{\mathrm{POX}}$ & Settling velocity of particulate dead organic matter & 1.5 & $\mathrm{~m} \mathrm{~d}^{-1}$ \\
$W$ & Wind velocity & $* * *$ & $\mathrm{~m} \mathrm{~s}^{-1}$ \\
$Z$ & Water depth & $* * *$ & $\mathrm{~m}$ \\
\hline
\end{tabular}

*In Table 3

**Calculated in GEM

***Model forcing

Table 3 Parameter values in the algal module (BLOOM) of the GEM model

\begin{tabular}{lllllllllll}
\hline ALGi & $\mathrm{e}_{\mathrm{ALGi}}$ & $\mathrm{s}_{\mathrm{N}, \mathrm{i}}$ & $\mathrm{s}_{\mathrm{P}, \mathrm{i}}$ & $\mathrm{s}_{\mathrm{Si}, \mathrm{i}}$ & $\mathrm{s}_{\mathrm{Chl}, \mathrm{i}}$ & $\mathrm{p}_{\mathrm{i}, 0}$ & $\mathrm{kt}_{\mathrm{p}, \mathrm{i}}$ & $\mathrm{m}_{\mathrm{i}, 0}$ & $\mathrm{kt}_{\mathrm{m}, \mathrm{i}}$ & $\mathrm{v}_{\mathrm{ALGi}}$ \\
\hline diat-E & 0.24 & 0.255 & 0.032 & 0.447 & 0.053 & 0.083 & -1.75 & 0.07 & 1.072 & 0.5 \\
diat-N & 0.21 & 0.07 & 0.012 & 0.283 & 0.01 & 0.066 & -2 & 0.08 & 1.085 & 1 \\
diat-P & 0.21 & 0.105 & 0.01 & 0.152 & 0.01 & 0.066 & -2 & 0.08 & 1.085 & 1 \\
flag-E & 0.25 & 0.2 & 0.02 & 0 & 0.023 & 0.09 & -1 & 0.07 & 1.072 & 0 \\
flag-N & 0.225 & 0.078 & 0.01 & 0 & 0.007 & 0.075 & -1 & 0.08 & 1.085 & 0.5 \\
flag-P & 0.225 & 0.113 & 0.007 & 0 & 0.007 & 0.075 & -1 & 0.08 & 1.085 & 0.5 \\
dino-E & 0.2 & 0.163 & 0.017 & 0 & 0.023 & 0.132 & 5.5 & 0.075 & 1.072 & 0 \\
dino-N & 0.175 & 0.064 & 0.011 & 0 & 0.007 & 0.113 & 4.75 & 0.08 & 1.085 & 0 \\
dino-P & 0.175 & 0.071 & 0.01 & 0 & 0.007 & 0.112 & 4.75 & 0.08 & 1.085 & 0 \\
Phaeo-E & 0.45 & 0.188 & 0.023 & 0 & 0.023 & 0.084 & -3.25 & 0.07 & 1.072 & 0 \\
Phaeo-N & 0.413 & 0.075 & 0.014 & 0 & 0.007 & 0.078 & -3 & 0.08 & 1.085 & 0.5 \\
Phaeo-P & 0.413 & 0.104 & 0.011 & 0 & 0.007 & 0.078 & -3 & 0.08 & 1.085 & 0.5 \\
\hline
\end{tabular}

conditions. In accordance with the aforementioned principle, the suitability of a type (its fitness) is determined by the ratio of its requirement and its growth rate. It can be shown mathematically that the principle by which each phytoplankton type maximises its own benefit effectively means that the total net production of the phytoplankton community is maximised. This makes it possible to use the computationally efficient Linear Programming technique (Danzig, 1963) to compute the phytoplankton biomasses according to the competition rules formulated for the module. Mathematically, this means that the optimisation process finds the combination of phytoplankton types that maximise the net growth subject to the following set of constraints.

- growth constraint: the biomass increase of any of the species groups cannot exceed the maximum net growth rate (production minus respiration) at actual temperature and light intensity. The relation between light intensity and growth efficiency (as a fraction of the maximum net growth rate) is included in the model as tabulated P-E curves, based on laboratory studies.

- mortality constraint: the mortality rate of any of the species groups cannot exceed the maximum mortality rate at actual temperature and salinity.

- light constraints: the total extinction of light by phytoplankton cannot exceed the threshold level where the light intensity becomes insufficient to maintain further net growth. The growth response to varying light intensities is implemented as tabulated data, based on laboratory studies. The tabulated response curve is converted to account for variable depth and day length.

- nutrient constraints: the total uptake of each of the nutrients $(\mathrm{N}, \mathrm{P}, \mathrm{Si})$ must not exceed the 
availability. The total available amount of a nutrient is defined as the sum of dissolved inorganic nutrient plus the amount of nutrients in phytoplankton.

Due to mortality, phytoplankton biomass is released partly as dead particulate organic matter and partly as inorganic nutrients in the water column, the latter representing autolysis. Different kinds of freshwater and marine phytoplankton can be modelled simultaneously. The uptake of nitrogen is done preferably as ammonium $\left(\mathrm{NH}_{4}{ }^{+}\right)$. When ammonium is depleted, the remainder of nitrogen uptake is done as nitrate. Most models require an explicit term to delimit the growth rate, forcing it to zero when a nutrient gets depleted. BLOOM does not require such a term since the Linear Programming procedure automatically stops the uptake when the concentration becomes zero. The growth rate in BLOOM declines step-wise as a nutrient gets depleted. When the E-types dominate, the net growth rate is high; then, it declines abruptly when a nutrient limited type takes over, and finally, it is put to zero when the nutrient is depleted.

\section{Extinction of light}

Light is simulated as total photosynthetic active radiation (PAR). Extinction of light by substances in the water is modelled as an exponential decrease of light intensity with depth according to the LambertBeer formula. The extinction coefficient is calculated as the sum of the extinction by inorganic suspended matter, particulate organic matter, phytoplankton (selfshading), dissolved humic substances (approximated by salinity) and background extinction. Each of the substances, including the phytoplankton species, is characterised by a specific extinction coefficient.

Decomposition of dead particulate organic matter

The decomposition rate of dead particulate organic matter (POM) in water is dependent on the nutrient stoichiometry of detritus, since bacteria need a supply of nutrients that is proportional to the supply of organic carbon. The decomposition rate of POM is highest for POM with a high nutrient content (expressed as N/C and $\mathrm{P} / \mathrm{C}$ ratios). Therefore, if the nutrient content is above a threshold value, a high decomposition rate is applied in the model. If the nutrient content is below another threshold, a low decomposition rate is applied. If the nutrient content is in between the two thresholds, the decomposition rate is linearly interpolated. The nutrient content of the most limiting nutrient $(\mathrm{N}$ or $\mathrm{P})$ determines the overall decomposition rate for POC, PON and POP. This approach was first applied in the freshwater model DBS as described by Van der Molen et al. (1994).

Particulate organic matter in the sediment is formed upon settling of phytoplankton and dead particulate organic matter. In the model, decomposition rate is affected only by temperature, which is assumed to be lower in the sediment than in the water. Remineralised inorganic nutrients are released back into the water column. In most GEM applications, the sediment is not included as a separate layer in the model grid. This means that only settled organic matter $(\mathrm{C}, \mathrm{N}, \mathrm{P}, \mathrm{Si})$ is simulated as a model variable, but nutrient concentrations and oxygen in porewater are not. Instead, remineralised nutrients are released directly back into the water column. The advantage of this simplification is the reduction of the number of grid cells and simulation time. The disadvantage is that processes that are in reality strongly non-linear, such as the release of ortho-phosphate from anaerobic sediments, cannot be taken into account properly. This disadvantage is particularly apparent in shallow coastal areas. In such shallow (often eutrophic) systems, a more sophisticated sediment module may have to be applied, which includes reduction \& oxidation as well as $\mathrm{P}$ adsorption and desorption processes in the sediment. This model improvement for shallow systems is currently being developed and tested.

\section{Nitrification and denitrification}

Nitrification and denitrification are modelled as simple first order processes, corrected for temperature and oxygen concentration in the water column. Nitrification rates decrease when the oxygen concentrations in the water drop below a critical level.

\section{Reaeration}

The difference between the actual oxygen concentration in the water and the saturation concentrations is reduced by the reaeration rate. The saturation concentration of oxygen is a function of temperature and salinity of the water. The reaeration rate in most 
GEM applications is a function of wind speed and water depth.

\section{Settling}

Settling in GEM is generally modelled as a net settling process with a settling velocity that is constant in time, and independent from turbulence and bottom shear stress. In 3D simulations, the effect of turbulence is accounted for by vertical dispersion between the water layers. Optionally, resuspension and the effect of bottom shear stress can be included. For each phytoplankton species and phenotype, a separate settling velocity is specified. For POM, one settling velocity is specified that is applied for all nutrient fractions (POC, PON, POP and $\mathrm{POSi}_{\mathrm{S}}$ ).

\section{Burial}

Particulate organic matter in the sediment is removed from the active sediment layer through burial to deeper sediment layers. These deeper sediment layers are not included in the model, and so in effect, the burial is a sink from the nutrient cycle in the model. The burial rate is a constant fraction of the particulate organic matter in the sediment throughout the year. The burial rate is a calibration parameter. It does not only represent the actual burial process, which is generally unknown for marine ecosystems, but also all other unknowns in the mass balance, including uncertainties on loading and transport across open boundaries.

\section{Filterfeeder processes}

Primary consumers are not simulated as real state variables in GEM. Instead, grazer biomass can be imposed to the model as a forcing condition (based on field data). The model then simulates the grazing effects of the imposed filterfeeder biomass. Five types of filterfeeders can be defined, both pelagic and benthic. The imposed biomass of primary consumers is adjusted by the model if availability of food (algae and detritus) is insufficient to support the imposed filterfeeder biomass, according to the specified filtering rates and metabolic coefficients. In order to ensure conservation of mass balance, nutrient concentrations are corrected in accordance with these adjustments in filterfeeder biomass. In case the filterfeeder module is applied for scenario simulations, a decision needs to be made whether or not to adjust its biomass function. This is usually done on the basis of expert knowledge taking observed grazer biomasses in various comparable water systems into account This approach, originally developed by Van der Molen et al. (1994), is considered as a first step in dynamic modelling of primary consumers.

At high densities of phytoplankton and detritus, the uptake of food is determined by the uptake rate. At low densities of phytoplankton and detritus, the uptake of food is determined by the filtration rate. Part of the uptake is egested as detritus. The egestion rate is a constant fraction of the uptake rate per type of food. The egestion fraction $\left(f_{\text {eg }}\right)$ may be different for each phytoplankton or detritus type. For benthic filterfeeders, the detritus is excreted to the sediment, and for pelagic filterfeeders, it is excreted to the water column. The composition of grazers as $\mathrm{C}: \mathrm{N}: \mathrm{P}$ ratio is constant over time. Therefore, the assimilated food should have the same C:N:P ratio as the grazers. The part of the uptake of the non-limiting nutrients that cannot be assimilated is excreted as detritus. Part of the food uptake is lost due to growth respiration. The remaining part is available for biomass increase of the filterfeeders. The realised biomass increase (or decrease) is given by the forcing function, but is constrained by maximum growth, maximum mortality and food availability. The food that is not used for biomass increase and organic matter associated with biomass decrease is all released as detritus. Uptake, filtration, growth, maintenance respiration, growth respiration and mortality are all affected by temperature. The temperature coefficients can be chosen differently for each process, but generally, the same temperature coefficient is used for each process.

\section{Applicability of the 'gem' model}

The GEM model has been successfully applied and validated in several projects in a range of different estuarine and coastal water systems. This section presents a number of selected case study simulations to illustrate the generic applicability of the model. It would be beyond the scope of the present paper to describe each of these case studies in great detail. They are introduced here as representative examples to demonstrate the success with which GEM was repeatedly applied in a range of different water systems and 
geographical areas without the need for continuous remaking of (new) models for each different system, site or objective, or for reparameterization of process parameters and state variables.

The 'goodness-of-fit' between these model results and associated field measurements was calculated using the 'cost function' (OSPAR, 1998; Radach \& Moll, 2006). The cost function is a mathematical function that gives a non-dimensional number, which is indicative of the 'goodness of fit' between two sets of data, in this case, model results and observations. It can be defined as the sum of the absolute deviations of the model values from the observations, normalised by the deviations of the observations for the chosen temporal and spatial range. Thus, it is a standardised, relative mean error. Cost function results for GEM applications have been calculated as

$C_{x}=\frac{\sum\left|M_{x, t}-D_{x, t}\right| / n}{s d_{x}} *\left((1-c)+c\left(1-r_{x}\right)\right.$

where $C_{x}$ is the normalised deviation per station, annual value, $M_{x, t}$ is mean value of the model results per station per month, ${ }^{2} D_{x, t}$ is mean value of the in situ data per station per month, $s d_{x}$ is standard deviation of the annual mean based on the monthly means of the in situ data ( $\mathrm{df}=11), n$ is 12 months, $c$ is 0.5 and $r_{x}$ is the correlation over time between $M_{x, t}$ and $D_{x, t}$ (OSPAR, 1998). The validation results were classified according to the following rating criteria (Radach \& Moll, 2006) for the cost functions (cf):

\begin{tabular}{lll}
\hline Rating & Condition & \\
\hline Very good & $0<\mathrm{cf} \leq 1$ & Standard deviations \\
Good & $1<\mathrm{cf} \leq 2$ & Standard deviations \\
Reasonable & $2<\mathrm{cf} \leq 3$ & Standard deviations \\
Poor & $3<\mathrm{cf}$ & Standard deviations \\
\hline
\end{tabular}

\section{Case 1-Veerse Meer}

The 'Veerse Meer' is a brackish coastal lake in the southwestern part of the Netherlands. This former

\footnotetext{
${ }^{2}$ For the Marmara Case study, in situ data were only available for two 60-day periods in a year, which were compared with corresponding 60-day periods of the model results.
}

estuary has been transformed into a non-tidal brackish lake, which is characterised by highly eutrophic conditions and seasonal blooms of the macroalga Ulva sp. (Malta \& Verschuure, 1997). The GEM model was used to study the effects of various water management alternatives on the water quality and ecology of the lake, in particular, the effects of water level changes and flushing scenarios (Kernkamp et al., 2002). Figure 2 presents the results of GEM model baseline simulations for the 'Veerse Meer' against monthly field observations (DONAR database, 1 station) for nutrient concentrations (nitrate, ammonium, phosphate and silicate), chlorophyll-a and dissolved oxygen for the period 1995-1999. Cost function results ranged from 0.235 to 2.330 (Table 4), indicating that these model results can objectively be classified as 'very good' for nitrate and ammonium, 'good' to 'very good' for phosphate and silicate, and 'reasonable' to 'good' for chlorophyll-a. These results demonstrate that the GEM model produced consistently good and acceptable results, in this case, for a range of different key parameters. Similarly, good results for a range of key parameters were obtained at other stations and in other case studies without the need for recalibration or reparameterisation.

\section{Case 2-North Sea}

The North Sea can be characterised as a coastal shelf sea with relatively shallow $(10-50 \mathrm{~m})$ coastal waters. Substantial river discharges result in large fluctuations and strong temporal and spatial gradients in salinity, suspended matter concentrations, nutrient concentrations and algal biomass. Over the years, GEM was applied to the North Sea system in a range of studies, including simulations to evaluate the impact of a proposed land reclamation scheme (Nolte et al., 2005), and as a screening tool for the Water Framework Directive (WFD) to demonstrate the potential impacts of reductions in nutrient loads (Blauw \& Los, 2004; Los \& Wijsman, 2007). Most recently, BLOOM/GEM applications to the southern North Sea were subjected to a detailed three-dimensional model validation (Los et al., 2008). Here, we present the results of a trend analysis of chlorophyll-a concentrations in the North Sea over a 24-year period (1975-1998) predicted by the GEM model and 
Fig. 2 Results of GEM baseline simulations for the "Veerse Meer" (surface water layers and bottom water layers) against actual field observations for nutrient concentrations (nitrate, ammonium, phosphate and silicate-all in $\mathrm{mg}^{-1}$ ), chlorophyll-a (in $\mu \mathrm{g}^{-1}$ ) and oxygen (in $\mathrm{mg}^{-1}$ ) for the period 1995-1999 (station: Soelekerkepolder)
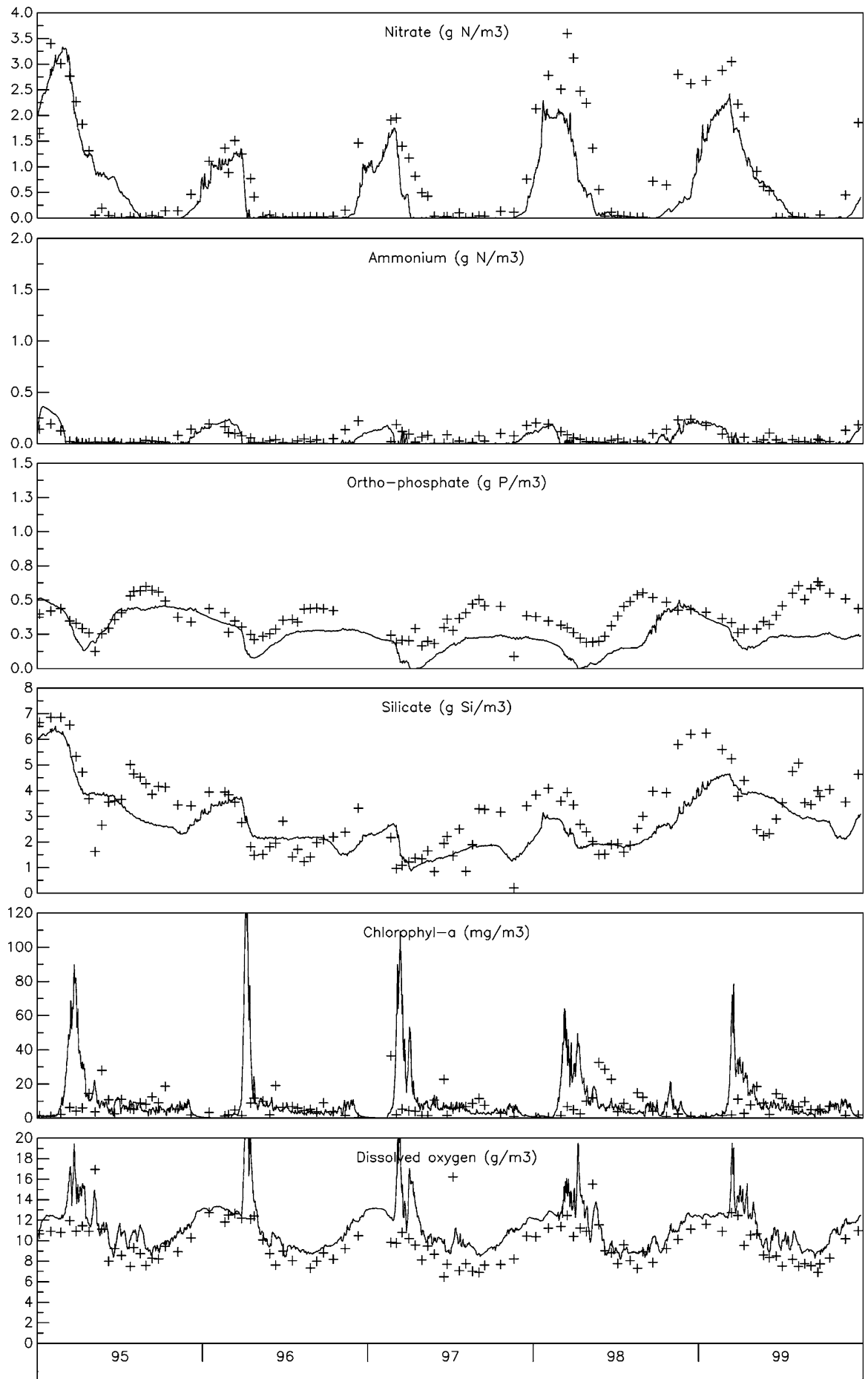

compared with field observations (DONAR database, monthly means measured over the same 24-year period) at six monitoring stations (Fig. 3 and Table 5). Figure 3 shows the results for the station
Terschelling $4 \mathrm{~km}$. The goodness-of-fit between model prediction and field measurements in the 1970s is not as perfect as in the 1980s and 1990s, and does not adequately reproduce their interannual 
Table 4 Cost function results for the Veerse Meer case (5 parameters, surface water, 1995-1999)

\begin{tabular}{llllll}
\hline Parameter & \multicolumn{5}{l}{ Year } \\
\cline { 2 - 6 } & 1995 & 1996 & 1997 & 1998 & 1999 \\
\hline $\mathrm{Chl}-a$ & 2.298 & 2.094 & 2.330 & 2.124 & 1.860 \\
$\mathrm{Si}$ & 1.026 & 0.809 & 1.179 & 0.778 & 1.006 \\
$\mathrm{PO}_{4}$ & 0.393 & 0.864 & 1.257 & 1.389 & 1.090 \\
$\mathrm{NO}_{3}$ & 0.265 & 0.441 & 0.480 & 0.249 & 0.235 \\
$\mathrm{NH}_{4}$ & 0.698 & 0.536 & 0.512 & 0.329 & 0.470 \\
\hline
\end{tabular}

variability. This may be related to the following two issues: [1] all model predictions were based on simulations with the same hydrodynamics (single, representative day) and same suspended sediment forcings (steady state, cosine-transformed for seasonality) (as described in Los \& Bokhorst, 1997), which may have reduced interannual variability in the model results; [2] there has been a shift from light limitation in the 1970s to nutrient $(\mathrm{P})$ limitation in the late 1980s and 1990s (see www.waterbase.nl). Apparently, the model performs better under P-limited conditions given the stoichiometric settings chosen in the model set-up. Cost function results for individual years and stations ranged from 0.287 to 1.348 (Table 5), indicating that the model results for chlorophyll-a remained 'good' (one station) to 'very good' (other stations) throughout all of these years. In order to illustrate that it is also possible to make reasonable predictions of the phytoplankton species composition using GEM (Los \& Blaas, in prep.), an example of detailed phytoplankton results is presented in Fig. 4, showing phytoplankton species composition as modelled in GEM versus field observations. The results of the 24-year analysis of chlorophyll-a demonstrate that the GEM model produces consistently good and acceptable results for this key parameter over many consecutive years without the need for recalibration or reparameterisation.

\section{Case 3-Venice Lagoon}

The Lagoon of Venice is a very shallow (average depth $1.1 \mathrm{~m}$ ), saline, subtropical, semi-enclosed estuarine lagoon system bordering the Italian city of Venice along the Mediterranean coast. The lagoon system covers an area of approximately $550 \mathrm{~km}^{2}$ and is characterised by eutrophic conditions and seasonal blooms of the macroalga Ulva sp.. Over the past two decades, the lagoon has gone through substantial changes in ecological quality and water quality as a result of changes in nutrient loads, sediment
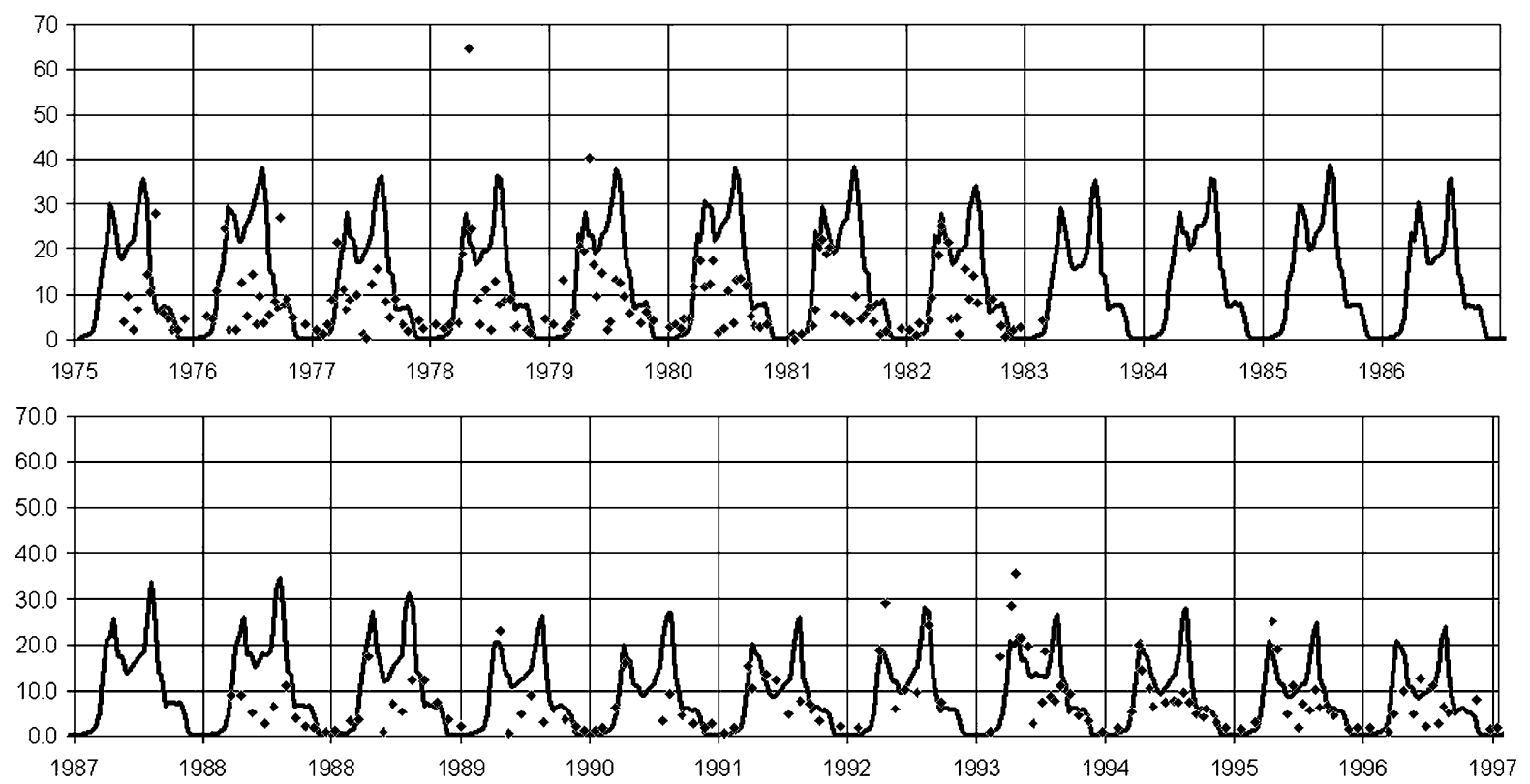

Fig. 3 Results of a trend analysis of chlorophyll-a concentrations (in $\mu \mathrm{g}^{-1}$ ) in the North Sea over a 24-year period (1975-1998) predicted by the GEM model in comparison with field observations (station: Terschelling $4 \mathrm{~km}$ ) 
Table 5 Cost function results for the North Sea case (chlorophyll-a, 24 years, 6 stations)

\begin{tabular}{|c|c|c|c|c|c|c|}
\hline \multirow[t]{2}{*}{ Year } & \multicolumn{6}{|l|}{ Station } \\
\hline & Noordwijk 4 & Noordwijk 10 & Noordwijk 20 & Noordwijk 70 & Terschelling 4 & Terschelling 10 \\
\hline 1975 & 0.314 & 0.353 & 0.714 & 1.347 & 0.751 & 0.744 \\
\hline 1976 & 0.315 & 0.347 & 0.704 & 1.348 & 0.903 & 0.730 \\
\hline 1977 & 0.309 & 0.348 & 0.699 & 1.341 & 0.775 & 0.726 \\
\hline 1978 & 0.311 & 0.366 & 0.710 & 1.340 & 0.718 & 0.723 \\
\hline 1979 & 0.321 & 0.353 & 0.714 & 1.343 & 0.869 & 0.703 \\
\hline 1980 & 0.321 & 0.347 & 0.712 & 1.344 & 0.923 & 0.715 \\
\hline 1981 & 0.319 & 0.359 & 0.710 & 1.343 & 0.889 & 0.711 \\
\hline 1982 & 0.318 & 0.359 & 0.703 & 1.342 & 0.743 & 0.731 \\
\hline 1983 & 0.305 & 0.352 & 0.688 & 1.341 & 0.656 & 0.727 \\
\hline 1984 & 0.312 & 0.366 & 0.710 & 1.344 & 0.829 & 0.690 \\
\hline 1985 & 0.313 & 0.356 & 0.708 & 1.344 & 0.872 & 0.697 \\
\hline 1986 & 0.305 & 0.351 & 0.691 & 1.343 & 0.719 & 0.725 \\
\hline 1987 & 0.301 & 0.360 & 0.680 & 1.336 & 0.621 & 0.673 \\
\hline 1988 & 0.315 & 0.359 & 0.693 & 1.341 & 0.641 & 0.720 \\
\hline 1989 & 0.301 & 0.345 & 0.661 & 1.341 & 0.600 & 0.698 \\
\hline 1990 & 0.304 & 0.355 & 0.644 & 1.336 & 0.441 & 0.660 \\
\hline 1991 & 0.305 & 0.376 & 0.656 & 1.335 & 0.514 & 0.667 \\
\hline 1992 & 0.308 & 0.410 & 0.653 & 1.332 & 0.431 & 0.693 \\
\hline 1993 & 0.299 & 0.381 & 0.664 & 1.333 & 0.521 & 0.658 \\
\hline 1994 & 0.304 & 0.347 & 0.615 & 1.335 & 0.423 & 0.693 \\
\hline 1995 & 0.311 & 0.409 & 0.668 & 1.333 & 0.456 & 0.690 \\
\hline 1996 & 0.290 & 0.420 & 0.645 & 1.329 & 0.402 & 0.641 \\
\hline 1997 & 0.297 & 0.425 & 0.622 & 1.327 & 0.395 & 0.684 \\
\hline 1998 & 0.287 & 0.403 & 0.634 & 1.330 & 0.417 & 0.638 \\
\hline
\end{tabular}

resuspension and anthropogenic perturbations (Sfriso et al., 2003). Large and lagoon-wide blooms of Ulva have not been observed anymore in the Lagoon of Venice over the last 15 years. The GEM model was used to study the potential effects of closure of mobile storm protection gates and reduction of nutrient loads on the environment, water quality and ecology of Venice Lagoon (Boon et al., 2006). Figure 5 presents the spatial results (horizontal dimension) of GEM model baseline simulations for chlorophyll-a concentrations (seasonal mean) for the entire Venice Lagoon in comparison with measured field data (Arresto campaign, 23 stations). This figure illustrates that the model was reasonably good in representing observed spatial variability in chlorophyll-a concentrations. Cost function results (based on the comparison of model results with monthly field measurements from the period June 1988 to June 1989) for chlorophyll-a, nitrate, ammonium and phosphate (Table 6) indicate that, in total, $78 \%$ of model results for these key parameters for all of 26 different stations can objectively be classified as 'good' to 'very good' and a further $13 \%$ as reasonable. Best results were obtained for nitrate and chlorophyll-a, with $92 \%$ and $73 \%$ of all model predictions falling in the categories 'good' and 'very good', respectively. The results of this analysis demonstrate that the GEM model produces consistently good and acceptable results for various key parameters throughout the horizontal (spatial) dimension without the need for recalibration or reparameterisation.

\section{Case 4-Sea of Marmara}

The Sea of Marmara is a deep $(>2,000 \mathrm{~m})$, oligotrophic, temperate, stratified coastal sea in Turkey. As 

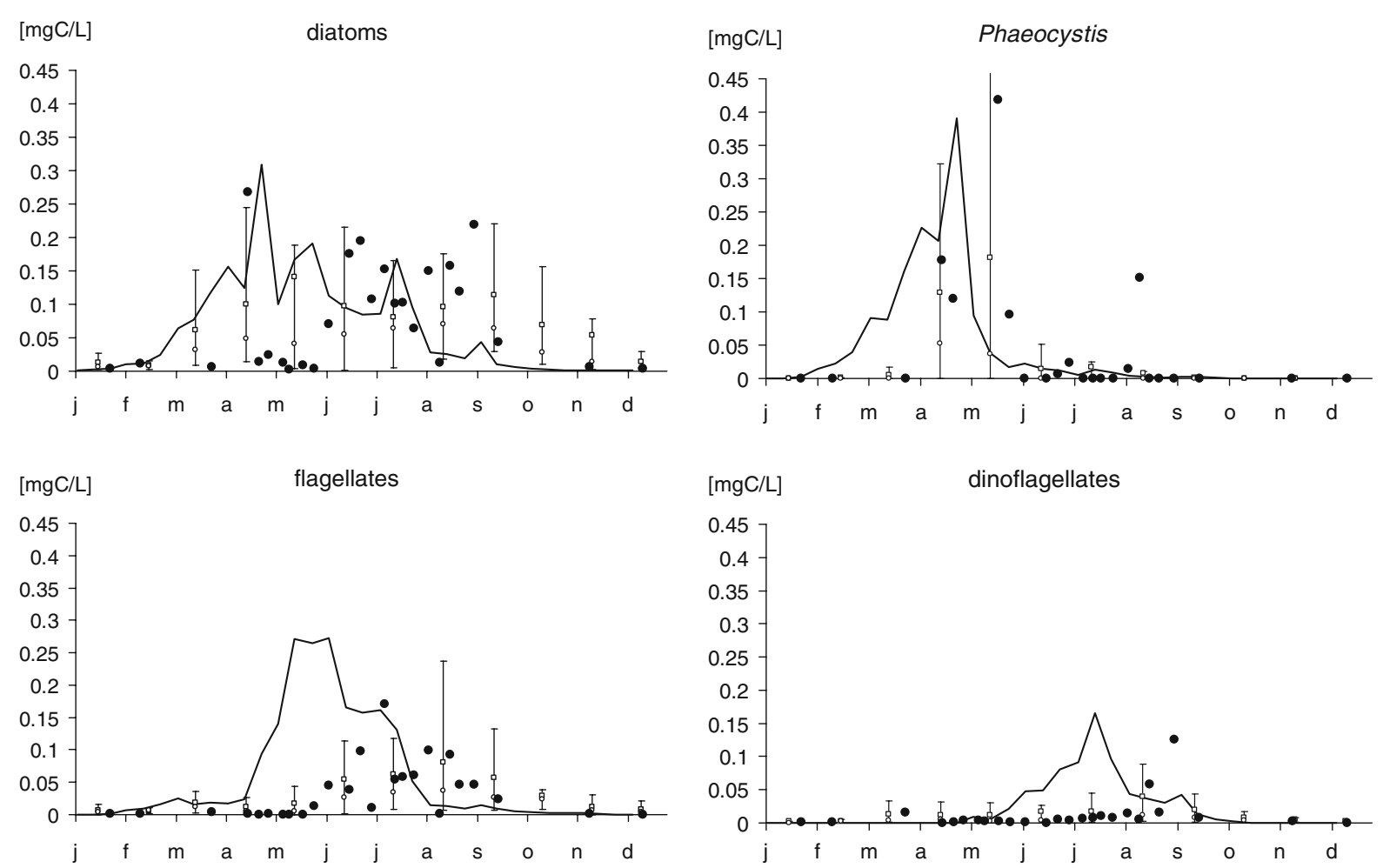

Fig. 4 Validation result for GEM algal species composition (1998 simulation) at station Noordwijk $10 \mathrm{~km}\left(\mathrm{~m} \mathrm{gC}^{-1}\right)$. Closed circles are measurements for 1998; bars indicate 90

percentile of measurements for the years 1991-2003, with open circles representing median and open squares representing mean values

part of a study for an environmental master plan and

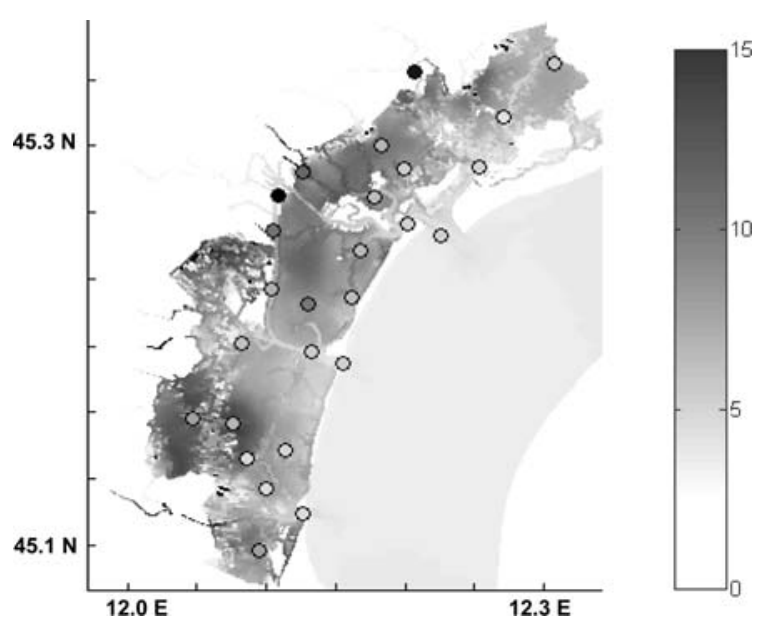

Fig. 5 Spatial results of GEM baseline simulation of chlorophyll-a in $\mu \mathrm{g}^{-1}$ (depth averaged) for Venice Lagoon showing seasonal mean values (April-September) of model results for summer 1988 in comparison with field measurements (Arresto Degrada monitoring data for April-September 1988). Marker colours of field data (circles) refer to the same scale as model output investment strategy for the Marmara Sea Basin, the GEM model was applied to simulate baseline water quality conditions and the implications of a number of predefined management scenarios (Smits, 2006). Figure 6 presents the results of an analysis of chlorophyll-a concentrations at different water depths (upper $60 \mathrm{~m}$ ) predicted by the GEM model (baseline 2003) for four different seasons, in comparison with in situ measurements at station MD56 (MEMPIS campaign). This figure illustrates that the model was reasonably good in representing observed seasonal variability in stratification patterns for chlorophyll-a. For the calculation of cost functions, model results for two 60-day periods (baseline 2003) were compared with available field observations (MEMPIS campaign, June and September 2005, 8 stations aggregated). Cost function results for nutrient concentrations (nitrate, phosphate and silicate), dissolved oxygen and chlorophyll-a at five different water depths (Table 7) indicate that, in total, $60 \%$ of model results can objectively be classified as 'good' to 'very 
Table 6 Cost function results for the Venice Lagoon case (4 parameters, 26 locations)

\begin{tabular}{|c|c|c|c|c|}
\hline \multirow[t]{2}{*}{ Location } & \multicolumn{4}{|l|}{ Parameter } \\
\hline & Chl- $a$ & $\mathrm{NO}_{3}$ & $\mathrm{NH}_{4}$ & $\mathrm{PO}_{4}$ \\
\hline Arresto 1 & 0.799 & 0.946 & 1.389 & 1.582 \\
\hline Arresto 2 & 3.479 & 0.690 & 1.460 & 1.130 \\
\hline Arresto 3 & 1.423 & 2.250 & 8.096 & 1.289 \\
\hline Arresto 4 & 2.041 & 1.943 & 3.666 & 1.578 \\
\hline Arresto 5 & 2.600 & 1.119 & 1.711 & 1.506 \\
\hline Arresto 6 & 1.019 & 0.339 & 1.186 & 1.252 \\
\hline Arresto 7 & 0.748 & 0.271 & 1.027 & 0.731 \\
\hline Arresto 8 & 0.441 & 1.155 & 1.376 & 1.571 \\
\hline Arresto 9 & 0.705 & 0.512 & 1.692 & 1.629 \\
\hline Arresto 10 & 0.504 & 0.927 & 1.342 & 1.782 \\
\hline Arresto 11 & 0.729 & 0.904 & 1.568 & 1.456 \\
\hline Arresto 12 & 1.303 & 0.602 & 1.628 & 2.331 \\
\hline Arresto 13 & 0.900 & 1.240 & 1.287 & 1.595 \\
\hline Arresto 14 & 2.024 & 0.546 & 2.137 & 1.332 \\
\hline Arresto 15 & 1.112 & 0.446 & 1.665 & 1.172 \\
\hline Arresto 16 & 3.285 & 0.656 & 2.813 & 3.364 \\
\hline Arresto 17 & 1.322 & 0.985 & 3.011 & 2.531 \\
\hline Arresto 18 & 0.813 & 1.093 & 1.842 & 1.882 \\
\hline Arresto 19 & 1.137 & 2.231 & 3.100 & 4.069 \\
\hline Arresto 20 & 1.058 & 1.472 & 4.779 & 2.805 \\
\hline Arresto 22 & 0.835 & 0.459 & 0.847 & 0.865 \\
\hline Arresto 23 & 3.209 & 0.936 & 2.335 & 1.893 \\
\hline Arresto 24 & 0.810 & 0.311 & 0.999 & 0.678 \\
\hline Arresto 25 & 0.607 & 0.496 & 0.724 & 1.235 \\
\hline Arresto 26 & 0.823 & 0.567 & 1.154 & 1.088 \\
\hline Arresto 27 & 2.007 & 0.638 & 2.596 & 1.520 \\
\hline Percentages & Very good & Good & Reasonable & Poor \\
\hline Chl- $a$ & 46 & 27 & 15 & 12 \\
\hline $\mathrm{NO}_{3}$ & 69 & 23 & 8 & 0 \\
\hline $\mathrm{NH}_{4}$ & 12 & 54 & 15 & 19 \\
\hline $\mathrm{PO}_{4}$ & 12 & 69 & 12 & 8 \\
\hline
\end{tabular}

good' and a further $12 \%$ as reasonable. GEM model results for chlorophyll-a, nitrate and dissolved oxygen in the deeper layers (40 and $50 \mathrm{~m}$ ) compared poorly with in situ data, but the cost function results were 'reasonable' to 'good' for silicate and 'very good' for phosphate at these depths. The overall results of this analysis demonstrate that the GEM model produces consistently good and acceptable results for various key parameters throughout the vertical dimension (depth) without the need for recalibration or reparameterisation.

\section{Discussion}

The present paper describes the set-up, application and validation of a GEM for estuaries and coastal waters. Since its development in the mid-1990s, the GEM model has been applied successfully in a range of consultancy and scenario studies, which have formed the basis for several major policy and management decisions and infrastructural developments in coastal zones in the Netherlands and worldwide. 
Fig. 6 Chlorophyll-a concentrations (in $\mu \mathrm{g}^{-1}$ ) at different water depths in the Sea of Marmara as predicted by the GEM model (baseline 2003) for four different seasons in comparison with field observations from 2005 (station: MD56)
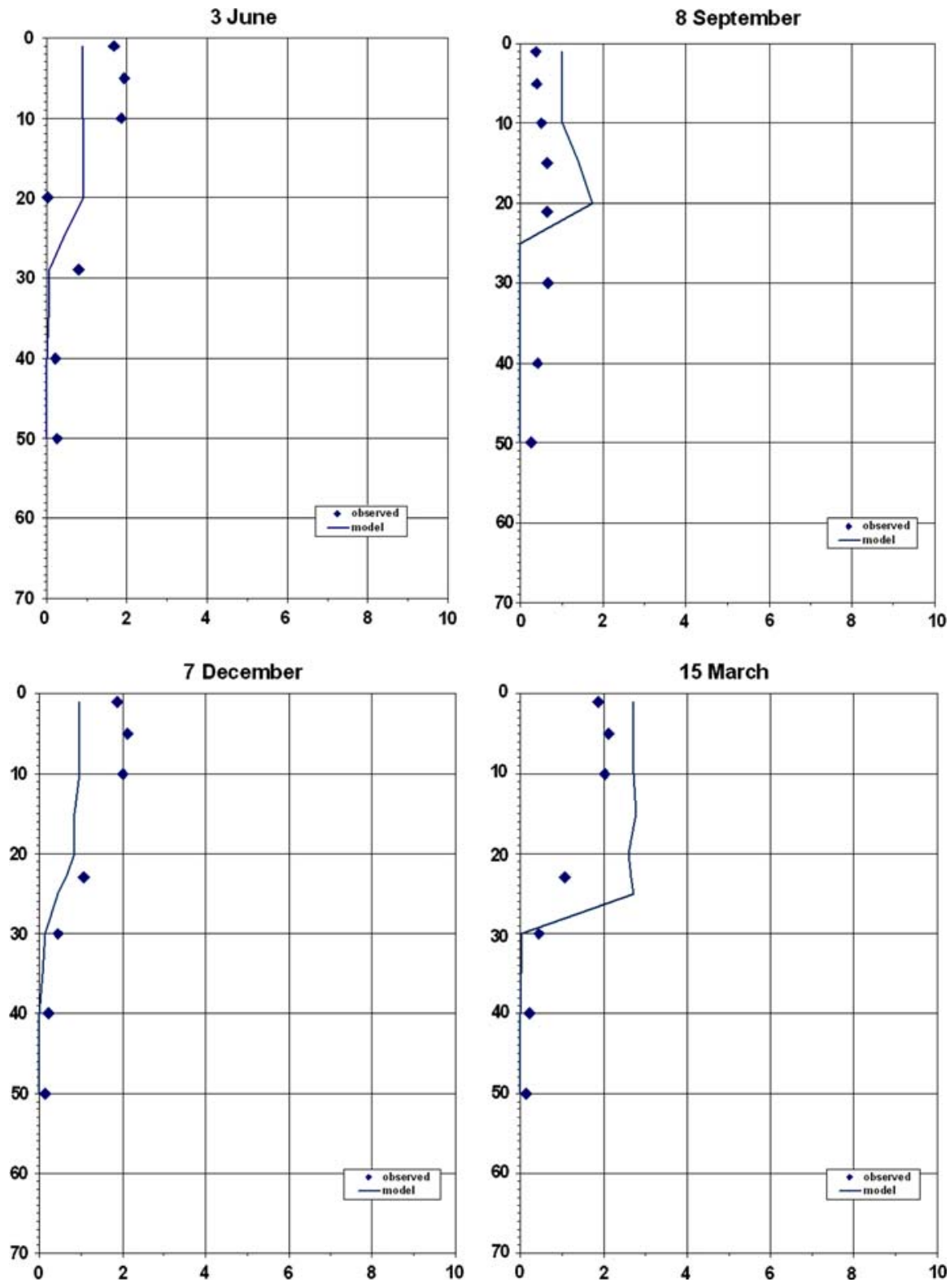

Table 7 Cost function results for the Sea of Marmara case (5 parameters, 5 depths)

\begin{tabular}{llllll}
\hline Parameter & \multicolumn{5}{l}{ Depth } \\
\cline { 2 - 6 } & 10 & 20 & 30 & 40 & 50 \\
\hline $\mathrm{Chl}-a$ & 0.408 & 3.136 & 1.821 & 4.618 & 8.235 \\
$\mathrm{Si}$ & 2.374 & 0.649 & 1.453 & 1.736 & 2.033 \\
$\mathrm{PO}_{4}$ & 1.412 & 0.468 & 0.499 & 0.955 & 0.910 \\
$\mathrm{NO}_{3}$ & 1.483 & 1.264 & 2.961 & 4.780 & 5.792 \\
Oxygen & 0.141 & 0.421 & 1.988 & 4.704 & 7.888 \\
\hline
\end{tabular}

Using a number of representative examples from case studies in which the GEM model was applied, the generic applicability of the GEM model was evaluated. Validation of the examples using the 'cost function' revealed consistent accuracy of the GEM model for various key parameters (Case 1), for both horizontal (Case 3) and vertical (Case 4) spatial dimensions, as well as temporal dimensions (seasonally and across years, Case 2) for a variety of estuarine and coastal water systems without the need for substantial reparameterisation. In addition, it is 
also possible to make reasonable predictions of the phytoplankton species composition using GEM (Fig. 4), which is further elaborated in another forthcoming paper (Los \& Blaas, in prep.).

In each of the four applications of GEM presented in this paper, model implementation for a new area usually required careful checking and improving physical forcings (loads, suspended matter, hydrodynamic and hydrological boundary conditions), which are typically area-specific. In addition, it was sometimes necessary to make minor adjustments to a few of the process parameters (in particular, to the closure terms denitrification and burial), resulting in minor differences in parameter settings between the four case studies. Dentrification rates applied in case studies $1-4$ were $0.003,0,0.05$ and 0.2 , respectively, in the sediment and $0,0.2,0.02$ and 0.15 , respectively, in the water column. Burial rates were only applied in the 2D cases of North Sea $\left(0.003\right.$ day $\left.^{-1}\right)$ and Venice Lagoon $\left(0.025 \mathrm{day}^{-1}\right)$. In the $3 \mathrm{D}$ cases (Veerse Meer and Sea of Marmara), settling rates for particulate variables were calibrated instead of burial, since settling in such deep permanently stratified systems has a similar effect as burial. Most other (esp. biological) model parameters, in particular, the settings for all of the key state variables for algae, were identical between the four case studies (Table 3). Table 2 shows the parameter settings used for the southern North Sea model, which is representative for the other model applications. Depending on the objectives of a particular study, the model setup can be extended with new processes specific to the particular site or application. For example, the phytoplankton group Phaeocystis was replaced by Ulva in the Venice Lagoon and Veerse Meer case studies, microphytobenthos was added in the Veerse Meer case study and $\mathrm{pH}$-dependent phosphorus adsorption was included in the Sea of Marmara and Veerse Meer case studies. For a more detailed description of the calibration and validation procedure for GEM \& BLOOM applications, see Los et al. (2008). A recent sensitivity analysis of GEM (Salacinska, 2008) using the Moris method (Saltelli et al., 2004), revealed that the Dutch coastal zone application of the GEM model is particularly sensitive to $\mathrm{Chl}$ :C ratios and light-related parameters (excl. extinction by $\mathrm{POM}$ ).

While in most cases GEM produced good to very good results, the goodness of fit between model results and field measurements was rather poor for some parameters in deeper layers (40 and $50 \mathrm{~m}$ ) of the oligotrophic Sea of Marmara and in very shallow (intertidal) areas of the Lagoon of Venice. For the Sea of Marmara, only a limited set of field observations was available for validation (due to low sampling frequency) and there was a mismatch between the timing of boundary forcings of the model (meteorological and hydrological data of 2003) and available field observations (2005), and so these results should be interpreted with some caution. For the Lagoon of Venice, poor model performance in very shallow areas of the lagoon (with extensive tidal flats) might be related to the periodic occurrence of anoxia. The formulations used for the sediment sub-model in GEM are not suited to account for such conditions.

Besides marine and brackish water systems, close relatives to the GEM model have also been applied successfully in a number of freshwater systems, such as the Dutch 'IJsselmeer' (Ibelings et al., 2003) and 'Veluwemeer' (Van der Molen et al., 1994) as well as 'Laguna de Bay' in the Philippines (Nolte \& Chua, 2003). For these freshwater applications, alternative algal parameters (not presented here) were used to reflect the different algal community in freshwater environments. With these additional (freshwater) algal parameters incorporated into GEM, the model is currently being applied in a study that compares (simultaneously) different freshwater and marine flushing scenarios for the Dutch 'VolkerakZoommeer'.

Limitations of the GEM model in its present state include application to very shallow dynamic environments with extensive tidal flats and periodic anoxia, due to inappropriate formulations used for the sediment sub-model in GEM. Application of GEM in deep and stratified oligotropic seas has been a challenge, producing poor results for some parameters at greater depths as shown in the Marmara case study. Performance of GEM in the Marmara case, however, was highly dependent on the accuracy of the underlying hydrodynamic model, which did not perfectly reproduce vertical stratification patterns. Since phytoplanktonic growth in GEM depends linearly on water temperature, while respiration and mortality follow the usual Arrhenius law, the ratio between growth and loss processes varies as a function of the temperature. During applications to tropical waters with higher temperatures compared to 
those for which the model was originally developed, we have found that loss rates could get unrealistically high relative to growth rates. As a practical solution, the temperature functions for all growth and loss processes had to be shifted in the model, while keeping the same relative proportion between them. Temperature coefficients of processes of the grazer module were modified in a similar way.

It should be stressed that these limitations are primarily and inherently related to the generic character of GEM (remaining unchanged in its settings and parameterisation between different case studies). Generally, such limitations can be overcome by further modifications to a specific model application, resulting in a more dedicated model. However, such dedicated models, while successful in their specific applications, tend to produce unsatisfactory results when applied (unchanged) in other case studies. Without compromising on its generic applicability, these issues and limitations are currently being addressed in further improvements to the GEM model.

Modelling of primary consumer processes remains a challenge to date. Existing grazer modules, such as ERSEM (and others), are currently being tested and validated for possible application and incorporation within the GEM model to allow for the modelling of higher trophic levels. Other developments within GEM that are currently underway include an improved module for sediment processes, including key geochemical processes (such as $\mathrm{P}$ adsorption and desorption) and more complex sedimention and resuspension formulations.

The current set-up of the GEM-model, including the explicit choices of which processes to include, allows for an optimal balance among ecological (process) resolution, model grid resolution and model run-time. ${ }^{3}$ Inclusion of microbial loops (incl. picoplankton etc.) and more detailed benthic processes (incl. improved mortality terms, differentiating complex burial processes), as is often done in various other (Dutch) ecological models, may yield greater insight into specific ecological processes, which can be useful when the model study has a more fundamental scientific focus. However, this invariably leads to exponential increases in model run-time, as

\footnotetext{
${ }^{3}$ Model run times in the four case studies ranged from approximately $1 \mathrm{~h}$ (Venice and Veerse Meer) to $11 \mathrm{~h}$ (North Sea) and $13 \mathrm{~h}$ (Sea of Marmara) per simulation.
}

a result of which the spatial resolution of the model is usually compromised. In most model applications designed to answer practical management questions, however, greater spatial resolution is usually required, while simplified closure terms for some of these more specific microbial and benthic processes often suffice, especially since field data on such processes are often lacking (Los et al., 2008).

Acknowledgments The authors would like to acknowledge the cooperation from all the people who participated in the development and various application studies of GEM, including Hanneke Baretta-Bekker, Marcel van der Tol, Peter Herman, René Jansen†, Piet Ruardij, Bert Brinkman, Johan de Kok, Johannes Smits, Leo Postma, Jan van Beek, Arno Nolte, Jos van Gils, Meinte Blaas, Xavier Desmit, Johan Boon and Tjitte Nauta. The development and validation of GEM was made possible by financial support of the National Institute of Coastal and Marine Management (RIKZ). Permission to make use of the monitoring data sets on Dutch waters (DONAR: www.waterbase.nl), Venice Lagoon (ARRESTO campaign) and Sea of Marmara (MEMPIS campaign) is kindly acknowledged. The Netherlands Ministries of Transport \& Public Works and Economic Affairs are thanked for their financial support within the framework of the WL I Delft Hydraulics R\&D Programme 2007, which enabled us to prepare this paper.

\section{References}

Airoldi, L. \& M. W. Beck, 2007. Loss, status and trends for coastal marine habitats of Europe. Oceanography and Marine Biology-Annual Review 45: 345-405.

Baretta, J. W., W. Ebenhoh \& P. Ruardij, 1995. The European Regional Seas Ecosystem model (ERSEM), a complex marine ecosystem model. Netherlands Journal of Sea Research 33: 233-246.

Blauw, A. N. \& F. J. Los, 2004. Analysis of the response of phytoplankton indicators in Dutch coastal waters to nutrient reduction scenarios. WL I Delft Hydraulics, technical report Z3844, December 2004, 145 pp.

Boon, J. G., X. Desmit \& M. Blaas, 2006. Ecological model of the Lagoon of Venice. Part II: Set-up, calibration and validation. WL I Delft Hydraulics, technical report Z3733, May 2006, $232 \mathrm{pp}$.

Brinkman, A. G., 1993. Biological processes in the Ecowasp ecosystem model. Institute for Forestry and Nature Research (IBN-DLO), IBN research report 93/6, ISSN: 0928-6896.

Danzig, G. B., 1963. Linear programming and extensions. Princeton University Press, Princeton, NJ.

De Kok, J. M., C. de Valk, J. H. T. M. Van Kester, E. de Goede \& R. E. Uittenbogaard, 2001. Salinity and temperature stratification in the Rhine plume. Estuarine, Coastal and Shelf Science 53: 467-475.

De Kok, J. M., R. Salden, I. D. M. Roozendaal, P. Blokland \& J. Lander, 1995. Transport paths of suspended matter along the Dutch coast. In Brebbia, C. A., L. Traversoni \& 
L. C. Wrobel (eds), Computer modelling of seas and coastal regions II. Computational Mechanics Publications, Southampton: $75-86$.

Delhez, E. J. M., P. Damm, E. D. De Goede, J. M. De Kok, F. Dumas, H. Gerritsen, J. E. Jones, J. Ozer, T. Pohmann, P. S. Rasch, M. D. Skogen \& R. Proctor, 2004. Variability of shelf-seas hydrodynamic models: lessons from the NOMADS2 project. Journal of Marine Systems 45: 39-53.

Devlin, M., M. Best \& D. Haynes, 2007. Implementation of the Water Framework Directive in European marine waters. Marine Pollution Bulletin 55: 1-2.

De Vries, I., R. N. D. Duin, J. C. H. Peeters, F. J. Los, M. Bokhorst \& R. W. P. M. Laane, 1998. Patterns and trends in nutrients and phytoplankton in Dutch coastal waters: comparison of time-series analysis, ecological model simulation and mesocosm experiments. ICES Journal of Marine Science 55: 620-634.

Fitz, H. C., E. B. DeBellevue, R. Costanza, R. Boumans, T. Maxwell, L. Wainger \& F. H. Sklar, 1996. Development of a general ecosystem model for a range of scales and ecosystems. Ecological Modelling 88: 263-295.

Gerritsen, H., J. G. Boon, T. Van der Kaaij \& R. J. Vos, 2001. Integrated modelling of suspended matter in the North Sea. Estuarine, Coastal and Shelf Science 53: 581-594.

Harris, G. P., 1986. Phytoplankton ecology. Chapman and Hall, London.

Ibelings, B. W., M. Vonk, H. F. J. Los, D. T. Van der Molen \& W. M. Mooij, 2003. Fuzzy modelling of cyanobacterial surface waterblooms: validation with NOAA-AVHRR satellite images. Ecological Applications 13: 1456-1472.

Kernkamp, H., G. Boot \& A. Nolte, 2002. Onderzoek naar de toekomstige waterkwaliteit en ecologie van het Veerse Meer. Studie naar het effect van het doorlaatmiddel en aanvullende maatregelen. Deel 1: Opzet en kalibratie hydrodynamisch en waterkwaliteitsmodel. WL I Delft Hydraulics, technical report Z3304, November 2002, 81 pp.

Klepper, O., W. M. Van der Tol, H. Scholten \& P. M. J. Herman, 1994. SMOES: a simulation model for the Oosterschelde ecosystem. Part 1: Description and uncertainty analysis. Hydrobiologia 282/283: 453-474.

Los, F. J., 2005. An algal biomass prediction model. In Loucks, D. P. \& E. Van Beek (eds), Water resources systems planning and management-an introduction to methods, models and applications. UNESCO, pp. 408-416.

Los, F. J. \& M. Bokhorst, 1997. Trend analysis Dutch coastal zone. In: New challenges for North Sea research, Zentrum fur Meeres- und Klimaforschung, University of Hamburg: 161-175.

Los, F. J. \& J. J. Brinkman, 1988. Phytoplankton modelling by means of optimization: a 10-year experience with BLOOM II. Verhandlungen der Internationalen Vereinigung für Theoretische und Angewandte Limnologie 23: 790-795.

Los, F. J., N. M. de Rooij \& J. G. C. Smits, 1984. Modelling eutrophication in shallow Dutch lakes. Verhandlungen der Internationalen Vereinigung für Theoretische und Angewandte Limnologie 22: 917-923.

Los, F. J., R. Jansen \& S. Cramer, 1994. MANS eutrophication modelling system. National Institute for Coastal and Marine Management/RIKZ, The Hague: 137.
Los, F. J., M. T. Villars, \& M. W. M. Van der Tol, 2008. A 3dimensional primary production model (BLOOM/GEM) and its applications to the (southern) North Sea (coupled physical-chemical-ecological model). Journal of Marine Systems. doi:101016/j.jmarsys.2008.01.002.

Los, F. J. \& J. W. M. Wijsman, 2007. Application of a validated primary production model (BLOOM) as a screening tool for marine, coastal and transitional waters. Journal of Marine Systems 64: 201-215.

Malta, E. J. \& J. M. Verschuure, 1997. Effects of environmental variables on between-year variation of Ulva growth and biomass in a eutrophic brackish lake. Journal of Sea Research 38: 71-84.

Moll, A. \& G. Radach, 2003. Review of three-dimensional ecological modelling related to the North Sea shelf system-Part 1: models and their results. Progress in Oceanography 57: 175-217.

Nolte, A., P. Boderie, \& J. van Beek, 2005. Impacts of Maasvlakte 2 on the Wadden Sea and North Sea coastal zone. Track 1: Detailed modelling research. Part III: Nutrients and Primary Production. WL I Delft Hydraulics, technical report Z3945, November 2005, 175 pp.

Nolte, A. \& G. Chua, 2003. Evaluation and future prospects of the Laguna de Bay Water Quality Model. Technical report for the "Sustainable Development of the Laguna de Bay Environment" project, WL I Delft Hydraulics, June 2003.

OSPAR, 1998. Report of the ASMO modelling workshop on eutrophication issues, 5-8 November 1996, The Hague, The Netherlands. OSPAR Commission Report, Netherlands Institute for Coastal and Marine Management/ RIKZ, The Hague, The Netherlands.

Ouboter, M. R. L., B. T. M. Van Eck, J. A. G. Van Gils, J. P. Sweerts \& M. T. Villars, 1998. Water quality modelling of the western Scheldt estuary. Hydrobiologia 366: 129142.

Radach, G. \& A. Moll, 2006. Review of three-dimensional ecological modelling related to the North Sea shelf system. Part II: Model validation and data needs. Oceanography and Marine Biology-Annual Review 44: $1-60$.

Reynolds, C. S., S. W. Wiseman, B. M. Godfrey \& C. Butterwick, 1983. Some effects of artificial mixing on the dynamics of phytoplankton populations in large limnetic enclosures. Journal of Plankton Research 5: 203-234.

Salacinska, K., 2008. Sensitivity analysis of the 2D application of the Generic Ecological Model to the North Sea. MSc thesis, Delft University of Technology, Delft, The Netherlands, $84 \mathrm{pp}$.

Saltelli, A., S. Tarantola, F. Campolongo \& M. Ratto, 2004. Sensitivity analysis in practice: a guide to assessing scientific models. Halsted Press, New York, NY, 2004.

Scholten, H. \& W. M. Van der Tol, 1994. SMOES: a simulation model for the Oosterschelde ecosystem. Part 2: Calibration and validation. Hydrobiologia 282/283: 453474.

Sfriso, A., C. Facca \& P. F. Ghetti, 2003. Temporal and spatial changes of macroalgae and phytoplanton in a Mediterranean coastal area: the Venice Lagoon as a case study. Marine Environmental Research 56: 617-636.

Smits, J., 2006. Environmental master plan and investment strategy for the Marmara Sea Basin. Water quality 
modelling of the Sea of Marmara: Model development and scenario simulations. WL I Delft Hydraulics, technical report Z3804.50, October 2006, 167 pp.

Soetaert, K. \& P. M. J. Herman, 1995a. Carbon flows in the Westerschelde estuary (the Netherlands) evaluated by means of an ecosystem model (MOSES). Hydrobiologia 311: 247-266.

Soetaert, K. \& P. M. J. Herman, 1995b. Nitrogen dynamics in the Westerschelde estuary (SW Netherlands) estimated by means of the ecosystem model MOSES. Hydrobiologia 311: 225-246.

Soetaert, K., P. M. J. Herman \& J. Kromkamp, 1994. Living in the twilight: estimating net phytoplankton growth in the Westerschelde estuary (The Netherlands) by means of an ecosystem model (MOSES). Journal of Plankton Research 16: 1277-1301.

United Nations Environment Programme (UNEP), 2006. Marine and coastal ecosystems and human well-being: a synthesis report based on the findings of the millennium ecosystem assessment. UNEP, 76 pp, Nairobi (see http://www.MAweb.org), ISBN: 92-807-2679-X.
Van der Molen, D. T., F. J. Los \& M. Van der Tol, 1994. Mathematical modelling as a tool for management in eutrophication control of shallow lakes. Hydrobiologia 275/276: 479-492.

Van de Wolfshaar, K. E., 2007. Beoordeling Generiek Ecologisch Model, GEM. Report summarising the outcome of an international audit of the GEM model. WL I Delft Hydraulics, technical report Z4267, January 2007, 47 pp.

Van Gils, J., 1998. The SOBEK processes editor: a flexible tool for tailor-made water quality modelling. In Babovic, V. \& L.C. Larsen (eds), Proceedings of the Third International Conference on Hydroinformatics, Copenhagen, Denmark. Balkema, Rotterdam, pp. 591-595.

Van Gils, J. A. G., M. R. L. Ouboter \& N. M. De Rooij, 1993. Modelling of water sediment quality in the Scheldt Estuary. Netherlands Journal of Aquatic Ecology 27: 257265.

WL I Delft Hydraulics, 2003. Delft3D-WAQ users manual. WL I Delft Hydraulics, Delft, The Netherlands. 